\title{
Multigenerational exposure to heat stress induces phenotypic resilience, and genetic and epigenetic variations in Arabidopsis thaliana offspring
}

Narendra Singh Yadav, Viktor Titov, Ivie Ayemere, Boseon Byeon, Yaroslav Ilnytskyy and Igor Kovalchuk*

Department of Biological Sciences, University of Lethbridge, Lethbridge T1K 3M4, Alberta, Canada.

*To whom correspondence should be addressed: igor.kovalchuk@uleth.ca

\begin{abstract}
Plants are sedentary organisms that constantly sense changes in their environment and react to various environmental cues. On a short-time scale, plants respond through alterations in their physiology, and on a long-time scale, plants alter their development and pass on the memory of stress to the progeny. The latter is controlled genetically and epigenetically and allows the progeny to be primed for future stress encounters, thus increasing the likelihood of survival. The current study intended to explore the effects of multigenerational heat stress in Arabidopsis thaliana. 25 generations of Arabidopsis (Col-0: 15d8) were propagated in the presence of heat stress. The stressed lineages $\mathrm{F} 2 \mathrm{H}$ (the $2^{\text {nd }}$ generation of the stressed progeny) and $\mathrm{F} 25 \mathrm{H}$ (the $25^{\text {th }}$ generation of the stressed progeny) have been studied to determine their response to heat stress at the stage of plant germination and maturity. Both the $\mathrm{F} 2 \mathrm{H}$ and $\mathrm{F} 25 \mathrm{H}$ stressed progenies did not show any significant differences from their parallel non-stressed progenies (F2C and $\mathrm{F} 25 \mathrm{C}$ ) at the germination stage but exhibited a higher tolerance at the mature- plant stage. Both stressed progenies exhibited the elevated frequency of homologous recombination $(\mathrm{HR})$ as compared to their parallel control progenies. A comparison of genomic sequences revealed that $\mathrm{F} 25 \mathrm{H}$ lineage had a three-fold higher number of mutations (SNPs and INDELs) as compared to the parallel (F25C) and parental (F2C) lineages, suggesting that heat stress induced genetic variations in the heat-stressed progeny. The $\mathrm{F} 25 \mathrm{H}$ stressed progeny showed a 7-fold higher number of non-synonymous mutations than the parental non-stress line which might lead to biological variations subjected to natural selection at the microevolution level. Gene Ontology Analysis revealed that SNPs were enriched mostly in unknown biological processes in all lineages, although processes such as response to stress and stimulus were enriched in the stressed lineage. However, the stressed lineage was underrepresented in the developmental processes, protein metabolism, cell
\end{abstract}


organization, and biogenesis. Methylome analysis revealed that the $\mathrm{F} 25 \mathrm{H}$ stressed progeny showed a lower global methylation in the $\mathrm{CHH}$ context than the control progenies which suggest that the reduction of methylation in the $\mathrm{CHH}(p<0.05)$ context might be a part of adaptation strategies to heat stress. The F25H lineage was different from the parental control lineage F2C by 66,491 differentially methylated positions (DMPs), but surprisingly, the parallel control (F25C) showed 80,464 DMPs compared to the parental control lineage, indicating that epigenetic variations were likely spontaneous in nature. The differentially methylated regions were enriched mostly in genes related to transcription and DNA dependent processes and DNA or RNA metabolism. Hierarchical clustering of these epimutations separated the heat stressed and control parental progenies into distinct groups which revealed the non-random nature of epimutations. Overall, our study showed that progenies derived from multigenerational heat stress displayed a notable stress memory in context to phenotypic, genotypic and epigenotypic resilience.

\section{Key words}

Multigenerational heat stress; Stress memory; Arabidopsis thaliana; Intergenerational inheritance; Transgenerational inheritance; Phenotypic resilience; Epigenetic variations; Genetic variations; Stressed lineage; Non-stressed lineage; SNPs; INDELs; Differentially methylated positions; Differentially methylated regions.

\section{Introduction}

Plants are continuously exposed to their surrounding environment which impacts their growth and agricultural yield (Wei et al. 2020). These interactions result in changes in gene expression and physiological and biomolecular responses that alter a plant's phenotype (Zheng et al. 2013; Wei et al. 2020). With the increased unpredictability of weather patterns and widespread environmental changes through global warming, many studies have started exploring the effects of multigenerational exposure of plants to environmental stressors such as heat, cold, drought, UV radiation, and other types of stresses. Species resilient to environmental changes have proven to be better suited for a new environment in comparison with their ancestors.

Plants respond to the environment through the adaptation and tolerance that ensure the survival of both the plant itself and its progeny (Huang et al. 2012). This phenomenon is feasible due to the plasticity of the plant genome and epigenome, and it evolves through the 
environmentally induced alterations and the development of phenotypic resilience (Nicotra et al. 2010; Kovalchuk and Kovalchuk 2012; Zhang et al. 2018; Lind and Spagopoulou 2018; Miryeganeh and Saze 2020). The phenotypic plasticity in response to environmental conditions is coordinated through the stress perception and activation of signalling pathways with cross-talks as well as the activation of responsive genes (Chinnusamy et al. 2004; Zheng et al. 2013). The adaptation to the environment is not restricted to changes in the physiology of the plant, but it also results in changes in the genome and epigenome (Gutzat and Scheid 2012; Zhang et al. 2018; Miryeganeh and Saze 2020)

Environmental stresses have an impact on the directly exposed generation as well as on their progenies via parental effects and/ or transgenerational effects (Herman and Sultan 2011). Parental effects are often referred to as intergenerational effects, they describe phenotypic changes in the immediate progeny of stressed plants. In contrast, transgenerational effects refer exclusively to changes in the phenotype of the progeny that is separated from the stressed progeny by at least one generation, thus representing real inheritance effects. However, many of the described intergenerational effects share their mechanisms with transgenerational effects, and many publications do not distinguish between the two, often referring to both as transgenerational (Perez and Lehner 2019). The RNA interference-related mechanisms can regulate the transgenerational inheritance of a specific chromatin or DNA modifications (Duempelmann et al. 2020). Very recently, Yang et al. (2020) have reported that the segregation of the MSHI RNAi transgene produces the heritable non-genetic memory in association with methylome reprogramming. The mshl reprogramming is dependent on functional HDA6 and DNA-methyltransferase MET1, and the transition to memory requires the RdDM pathway. This system of phenotypic plasticity may play a vital role for plant adaptation to the changing environment (Yang et al. 2020).

While the exact mechanisms of stress adaption and transgenerational stress memory remain unclear; the understanding of these processes has far-reaching implications for plant breeding, genetic engineering, and the development of stress tolerant crops (Bilichak and Kovalchuk 2016; Zhang et al. 2018). A substantial number of studies demonstrate the ability to maintain the memory of stress exposure throughout ontogenesis and transmit this memory to the following generation (Boyko and Kovalchuk 2010; Suter and Widmer 2013; Bilichak and Kovalchuk 2016; Ramírez-Carrasco et al. 2017; Zheng et al. 2017). For example, transgenerational stress memory of iron (Fe) deficiency was reported in Arabidopsis thaliana where the frequency of somatic homologous recombination events, DNA strands breaks and TFIIS-like gene expression increased for one generation when plants were grown under Fe 
deficiency (Murgia et al. 2015). In rice, Ou et al. (2012) reported transgenerational inheritance of modified DNA methylation patterns in three successive generations with an enhanced tolerance to heavy metal stress. Moreover, transgenerational inheritance of stress memory has been shown in multiple off-spring generations of Arabidopsis thaliana with or without the maintenance of the stress conditions (Groot et al. 2016). Higher reproductive outputs such as the number of flowers and seeds per plant were reported after exposures to heat treatment across multiple generations compared with the offspring grown in unheated environments (Whittle et al. 2009). Similarly, transgenerational effects in response to heat stress were reported over three generations in other model organisms like Artemia (Norouzitallab et al. 2014) and Caenorhabditis elegans (Gouvêa et al., 2015). RamírezCarrasco et al. (2017) showed that stress priming via beta-aminobutyric acid induced resistance to Pseudomonas syringae infection in the common bean over at least two generations.

The mechanism of stress memory most likely includes genetic and epigenetic changes, with the latter ones being more prevalent. The genetic variations within species are identified as Single Nucleotide Polymorphisms (SNPs) and Insertions and Deletions (INDELs). SNPs represent the difference at a single nucleotide base within individuals of the same species which may cause the phenotypic diversity such as flowering time adaptation, the colour of plant fruit and tolerance to various biotic and abiotic factors (Vidal et al. 2012; Jang et al. 2015). In the case of epigenetics, phenotype traits are transferred to the progeny without changes in gene sequence (Eichten et al. 2014; Bilichak and Kovalchuk 2016; Zheng et al. 2017; Lind and Spagopoulou 2018; Miryeganeh and Saze 2020). Epigenetic regulation consists of three primary mechanisms - DNA methylation, histone modifications and small RNAs (sRNAs) expression. DNA methylation is the most established gene expression modulation utilized by plants for changing their phenotypes in stressful environment, which acts as a complementary mode of transferring heritable information (Mirouze and Paszkowski 2011; Schmitz et al. 2011). Many recent reports have demonstrated that plant DNA methylation can be altered at individual loci or across the entire genome under environmental stress conditions (Zhang et al. 2018 and reference therein). In rice, the naturally occurring variations in DNA methylation are well studied epimutations at single gene loci that have been shown to result in heritable morphological variations without altering the DNA sequence of rice genes (Zheng et al. 2017; Miura et al. 2009). The chillinginduced loss of tomato flavor is associated with changes in DNA methylation, including the 
RIN gene (Zhang et al. 2016). In Arabidopsis, quantitative resistance to clubroot infection is mediated by transgenerational epigenetic variations (Liégard et al. 2019).

Epimutations occurring in response to environmental stress are reversible, thereby serving as a critical function in the adaptation of plants to their environment. Changes in DNA methylation are the most well-studied modifications; they include differentially methylated positions (DMPs) where single cytosines are involved and differentially methylated regions (DMRs) where multiple cytosines in a given region (arbitrarily defined) are considered. DMPs and DMRs are specific epimutations that can be associated with the epigenetic inheritance of stress response (Zhang et al. 2018). Exposure to environmental factors can induce changes in the specific DMRs, which suggest the existence of the exposure specific DMRs (Haque et al. 2016; Manikkam et al. 2012). Some examples include DMRs associated with the differentially expressed genes in the regulation of the Arabidopsis thaliana immune system against Pseudomonas syringae (Dowen et al. 2012) and the location-specific DMRs at specific cis-regulatory sites in the Hevea brasiliensis tree crop in response to cold and drought stresses (Uthup et al. 2011). Applying these genetic and epigenetic variation analyses to plants exposed to stressed conditions, we can decipher whether the exhibited phenotype is a result of either genetic or epigenetic variations. It will also enable us to define how each genetic/ epigenetic modification contributes to phenotypic differences (Lane et al. 2014).

Previous research from our laboratory demonstrated that the progeny of plants exposed to various abiotic and biotic stressors exhibited changes in phenotypic traits such as leaf size and number, flowering time, and seed size (Boyko et al. 2010; Bilichak et al. 2012; Migicovsky et al. 2014). The progeny of stressed plants also displayed a certain degree of stress tolerance and cross-tolerance lasting for one or two generations without the maintenance of stress factor (Rahavi and Kovalchuk 2013; Migicovsky et al. 2014). Our previous studies also demonstrated changes in the genome - rearrangements at the resistance genes loci in the progeny of plants infected with tobacco mosaic virus (Boyko et al. 2007) and in the epigenome - changes in DNA methylation and histone modifications in the progeny of plants exposed to various stresses such as salt, heat, cold, UVC and viral infection (Boyko et al. 2007 (viral); Bilichak et al. 2012 (salt); Migicovsky and Kovalchuk, 2013; Migicovsky and Kovalchuk, 2013 (cold); Migicovsky et al. 2014 (heat)). Although these studies did not explicitly analyse the mechanism of transgenerational inheritance, they proposed the role of epigenetic factors and demonstrated genetic changes relevant to stress exposure (Boyko et al. 2010; Boyko and Kovalchuk 2010; Bilichak et al. 2015). 
Based on studies conducted in our lab and several other labs, we hypothesized that multigenerational exposure to heat stress would be better in conveying stress memory on to the progeny than single generation stress. We also hypothesized that the plant population propagated under multigenerational heat stress would show a broader genotypic and epigenotypic diversity compared with the population of plants propagated without stress. We predicted that the multigenerational stressed lineages would exhibit the stress-specific features of genetic and epigenetic inheritance.

In this study, we have shown the ability of Arabidopsis plants to form heat stress memory using the repeated heat stress exposure over consecutive generations. 25 generations of Arabidopsis (Col-0: 15d8) were propagated in the presence of heat stress. The F2H and F25H generations of stressed progenies were compared with the F2C and F25C control progenies for heat stress resilience and the presence of stress-induced genetic and epigenetic changes. Therefore, the following questions have been addressed: Do the progeny of plants exposed to multigenerational heat stress exhibit a better response to heat stress than the progeny of a single-generation exposure to stress? Do genetic/epigenetic changes occur at a higher rate in the heat-stressed progenies compared with the progenies of non-stressed plants? Does a random or directional trend exist in the pattern of genetic and epigenetic variations induced by heat stress? And finally, can the biologically-enriched pathways associated with these genetic and epigenetic variations be predicted?

\section{Materials and Methods}

\section{Parental generation and progeny plants}

Plant seeds used for the parental generation $(\mathrm{P})$ were obtained from single homozygous Arabidopsis thaliana (Columbia ecotype, Col-0) plant transgenic for the luciferase (LUC) recombination reporter gene (15d8 line) carrying a copy of a direct repeat of the luciferase recombinant transgene. All parental plants were grown to flower, and seeds were collected and pooled from approximately 16-20 plants. The subsequent progeny plants were obtained from these seeds; specifically, $\sim 100$ seeds were sown, and $\sim 20$ randomly selected plants were either exposed to heat or grown under normal conditions, and then they started setting seeds, thus forming the next generation. The same process was repeated consecutively until twenty-five generations representing the progeny plant lines were obtained. The seeds were kept in storage under dry conditions at room temperature.

The progeny plants obtained from the progeny seeds through the process described above for every generation had two groups: the stressed $(\mathrm{H})$ and non-stressed groups $(\mathrm{C})$ 
which acted as the parallel control group within each generation. The ' $\mathrm{H}$ ' group included the heat-stressed progenies assisting in determining the potential role of responses to multigenerational heat stress exposure, while the ' $C$ ' group included plants grown under normal conditions acting as a parallel control.

All progeny plants representing the ' $\mathrm{H}$ ' group were heat treated during the development at $42^{\circ} \mathrm{C}$ for four hours per day for four consecutive days starting at day 10 post germination, and they were grown to flower and mature seed development. Seeds were then collected and propagated to obtain the next successive generation, thus creating 25 generations of the heat stressed ' $\mathrm{H}$ ' group with three biological replicates in each generation. On the other hand, the progeny plants representing the ' $C$ ' group were not heat stressed, they were grown under normal conditions and allowed to flower and mature seed development. Seeds were then collected and propagated to obtain the next successive generation, thus creating 25 generations of the non- stressed ' $C$ ' group with two biological replicates in each generation.

In summary, each generation had two replicates of ' $\mathrm{C}$ ' and three replicates of ' $\mathrm{H}$ ' plant lines, totalling five groups per generation.

\section{Preparation and growing plant seeds}

The seeds from the aforementioned parental, parallel and stressed progenies were kept at $4{ }^{\circ} \mathrm{C}$ for seven days to initiate stratification, then they were planted in all-purpose potting soil prepared with water containing a generic fertilizer (Miracle-Gro, Scotts Canada.) made to field capacity in $4 \times 4$ pots. The moist soil containing plant seeds was further stratified for 24 hours at $4^{\circ} \mathrm{C}$. Plants were grown in a growth chamber (BioChambers, Manitoba, Canada) at $22^{\circ} \mathrm{C}$ under the extended day conditions of 16 hours light and 8 hours dark $\left(18^{\circ} \mathrm{C}\right)$. Approximately three days post germination (dpg), plant seedlings were transplanted into individual pots containing a 9:1 ratio of soil to vermiculite composition prepared with a fertilizer. Pots containing plants were placed in trays measuring $\sim 25 \mathrm{~cm}$ x $50 \mathrm{~cm}$ and watered from below. This gave a group of a total of eight (8) plants per pot and two pots per sample, resulting in approximately sixteen (16) plants per treatment group.

\section{Lineages used for heat stress phenotyping}


The lineages F2C (parental control), F2H (the $2^{\text {nd }}$-generation stressed progeny), F25H (the $25^{\text {th }}$ generation stressed progeny) and F25C (the parallel control progeny) were used for study.

\section{Phenotypic analysis under heat stress treatment}

The F2H and F25H stressed progenies were studied for heat stress memory and response. The response to heat stress was determined at the germination or mature plant stage. For heat stress experiment, three biological replicate lines were used for the stressed progeny, and two biological replicate lines were used for the parallel control progeny. At germination level, heat stress was applied at $45^{\circ} \mathrm{C}$ for $6 \mathrm{~h}$, just after 4-days of stratification at $4^{\circ} \mathrm{C}$. The five biological replicates were used in one independent experiment. The percentage of germination and the speed of germination were qualitatively and quantitatively evaluated between the groups. To test the heat stress phenotype at the mature plant stage, the plants were grown under normal condition, and heat stress was applied just before bolting for $96 \mathrm{~h}$ at $40^{\circ} \mathrm{C}$. The qualitative and quantitative assessment has been done after 10-days of recovery. Phenotype changes such as thesurvival rate, fresh and dry weights of the survived plants were measured.

\section{The analysis of homologous recombination frequency (HRF)}

The Arabidopsis thaliana \#15d8 line transgenic for the luciferase (LUC) recombination reporter gene enables the analysis of HRF. Recombination events occur via the rearrangement between two homologous inactive copies of the luciferase transgene. HR events are analysed by scoring bright sectors on a dark background with a CCD camera after spraying with luciferin (Ilnytskyy et al. 2004). These sectors represent cells in which recombination events occurred. For the HRF analysis, we used at least 50 three-week-old plants per line. HRF was calculated by relating the number of events to the total number of plants scored. Each experiment was repeated at least twice.

\section{Lineages used for Whole Genome Sequencing (WGS) and Whole Genomic Bisulfite Sequencing (WGBS)}

The parental F2C plants and the progenies of (F25H and F25C) plants were grown to about 21 days post germination (dpg), rosette leaves tissues were harvested from individual plants 
and snap frozen in liquid Nitrogen, then they were stored at $-80^{\circ} \mathrm{C}$ for DNA extraction for sequence analysis. The total genomic DNA was extracted from approximately $100 \mathrm{mg}$ of leaf tissue homogenized in liquid Nitrogen using a CTAB protocol. From each generation, five individual plants were sequenced, resulting in a total of fifteen samples.

\section{Whole Genome Sequencing and Whole Genomic Bisulfite Sequencing}

The isolated genomic DNA was used for both whole genome sequencing (WGS) and whole genome bisulfite sequencing (WGBS) (Illumina) to assist in identifying the genomic and epigenomic (associated with changes in DNA methylation) profiles and variations. The data obtained were analysed using several toolkits found in the methylKit package.

WGBS allows for the investigation of genome-wide patterns of DNA methylation at a single-base resolution. It involves the sodium bisulfite conversion of unmethylated cytosine into uracil, with the resulting cytosine residues in the sequence representing methylated cytosine in the genome which is then mapped to a reference genome (Susan et al. 1994). Binomial tests were applied and used to determine the observed methylation frequency against the bisulfite conversion reaction, and the percentage of methylation levels were calculated at each base (Schultz et al. 2012).

\section{The computation and analysis of WGBS data}

Raw sequencing reads were quality controlled and trimmed using Trim Galore software (version 0.4.4). The trimmed reads were then aligned to the TAIR10 reference genome using the bisulfite mapping tool Bismark (Krueger and Andrews 2011). The methylated cytosines (Cs) were extracted from the aligned reads with the Bismark methylation extractor on default parameters followed by the computation of methylation frequency using the $\mathrm{R}$ package software, methylKit. The percentage of methylation was calculated by counting the frequency ratio of Cs divided by reads with $\mathrm{C}$ or a $\mathrm{T}$ at each base and computed at bases with coverage $\geq 10$ (Akalin et al. 2012).

The percentage $(\%)$ of methylation $=\{$ Frequency of $C \div$ read coverage $\} \times 100$

Common bases covered across all samples were extracted and compared, and the differential hyper- and hypo- methylated positions in each chromosome were extracted. The differentially methylated positions (DMPs) overlapping with genomic regions were assessed (in the preference of promotor $>$ exon $>$ intron), and the average percentage of methylation of 
DMPs around genes with the distances of DMPs to the nearest transcription start sites (TSSs) were also calculated.

The annotation analysis was performed with genomation package within methylKit to obtain a biological understanding of genomic intervals over the pre-defined functional regions such as promoters, exons, and introns (Akalin et al. 2014). The functional commentary of the generated gene expression profiles was performed using the SuperViewer tool with Bootstrap to show the difference between samples (Provart and Zhu 2003). Hierarchical clustering of samples was used to analyse similarities and detect sample outliners based on percentage methylation scores and a possible molecular signature. Principal Component Analysis (PCA) was utilized for variations and any biological relevant clustering of samples. Scatterplots and bar plots showing the percentage of hyper-/hypomethylated bases, the overall chromosome and heatmaps were used to visualize similarities and differences between DNA methylation profiles.

\section{The Differentially Methylated Regions (DMRs)}

A comparison of differential DNA methylation levels between samples reveals the locations of significant differential changes in the epigenome. The obtained information of DMRs was investigated over the predefined regions in all contexts: $\mathrm{CG}, \mathrm{CHG}$, and $\mathrm{CHH}$ for $100 \mathrm{bp}$ and $1000 \mathrm{bp}$ tiles across the genome to identify both stochastic and treatment-associated DMRs (Akalin et al. 2012).

The differential hyper-/hypo- methylated regions were extracted and compared across the samples. By default, DMRs were extracted with q-values $<0.01$ and the percentage of methylation difference $>25 \%$. The differential methylation patterns between the sample groups and the differences in methylation events of per chromosome were also extracted. The methylation profiles of the sample groups used were $\mathrm{F} 25 \mathrm{H}$ versus $\mathrm{F} 2 \mathrm{C}, \mathrm{F} 25 \mathrm{H}$ versus F25C, and F25C versus F2C. In summary, the sliding windows of $100 \mathrm{bp}$ and $1000 \mathrm{bp}$ were considered for both DMRs and DMPs, and extractions were made based on at least $25 \%$ and $50 \%$ differences (q-values $>0.01$ ) to assess the significant differences among samples.

\section{The computation and analysis of genome sequence data}

Raw sequencing reads were trimmed and aligned to the TAIR10 reference genome as described in WGBS data analysis; the only difference was that the duplicates were marked using Picard tool. Local realignments around SNPs and INDELs were performed using GATK (genome analysis toolkit) which accounts for genome aligners and mapping errors and 
identifies the consistent regions that contain SNPs and INDELs. The resulting reads were quality controlled with Haplotype scores and variant sample sites were called individually and jointly using the HaplotypeCaller with GATK. The sites marked as those that had a lowquality score by GATK were filtered out and used. The effects of variants in the genome sequences were classified using the SnpEff program (Cingolani et al. 2012).

Similar to the computational analysis of WBGS, the toolkits used the included genomation to obtain a biological understanding of genomic intervals and the Functional Classification SuperViewer to create gene expression profiles and show the difference between samples. The genes nearest to the non-overlapping SNP and INDEL sites were annotated.

\section{Statistical Analysis and Quality Control Values}

The mapped reads were obtained with a quality score of $<30$, the differential hyper- and hypomethylated bases were extracted with q-values $<0.01$ and the percentage of methylation difference larger than $25 \%$ in methylKit. The Heatmaps of differentially methylated bases were quantified at q-values $<0.01$, and the percentage of methylation difference was more significant than $50 \%$. The distances of DMPs to the nearest TSSs obtained from genomation at both $>25 \%$ and $>50 \%$ of methylation change. The distance between TSSs and DMPs was extracted within +/- 1000 bp and annotated at DMPs >50\% methylation difference. DNA methylation profiles obtained from the melthylKit used the obtained pairwise correlation coefficients of methylation levels (in \%) and the 1-Pearson's correlation coefficients for hierarchical clustering of samples. Logistic regression and Fisher's exact test were used for the determination of differential methylation with calculations of q-values and the BenjaminiHochberg procedure for the correction of $\mathrm{p}$-values. The T-test for mean difference between groups was calculated with p-values $<0.05$. The results of global genome methylation were plotted using Microsoft Excel (MS), and the output graphs from each corresponding program were used. The phenotypic data were analysed by the unpaired $t$-test with Welch's correction using GraphPad Prism version 8.4.2 for Windows. The data were shown as mean \pm SD. A $p$ value less than $0.05(p \leq 0.05)$ was considered statistically significant.

\section{Results}

The progeny of stressed plants showed the heat stress tolerance phenotype 
The $\mathrm{F} 2 \mathrm{H}$ and $\mathrm{F} 25 \mathrm{H}$ progenies were studied for heat stress memory and response. The response to heat stress was determined at the germination or mature-plant stage. The $\mathrm{F} 25 \mathrm{H}$ stressed progeny did not display any significant difference in germination index represented by the speed of germination and the total germination percentage (Figure 1A). We could not check the heat response at the germination level in the $\mathrm{F} 2 \mathrm{H}$ stressed progenies due to a very low germination efficiency ( 5\%; seeds were very old). Under heat stress, at the matureplant stage, both the F2H and F25H stressed progenies survived better than their respective non-stressed parallel control progenies (Figure 1B). However, F25H showed a slightly higher survival than F2H, but it was not statistically significant (Figure 1B). 
A
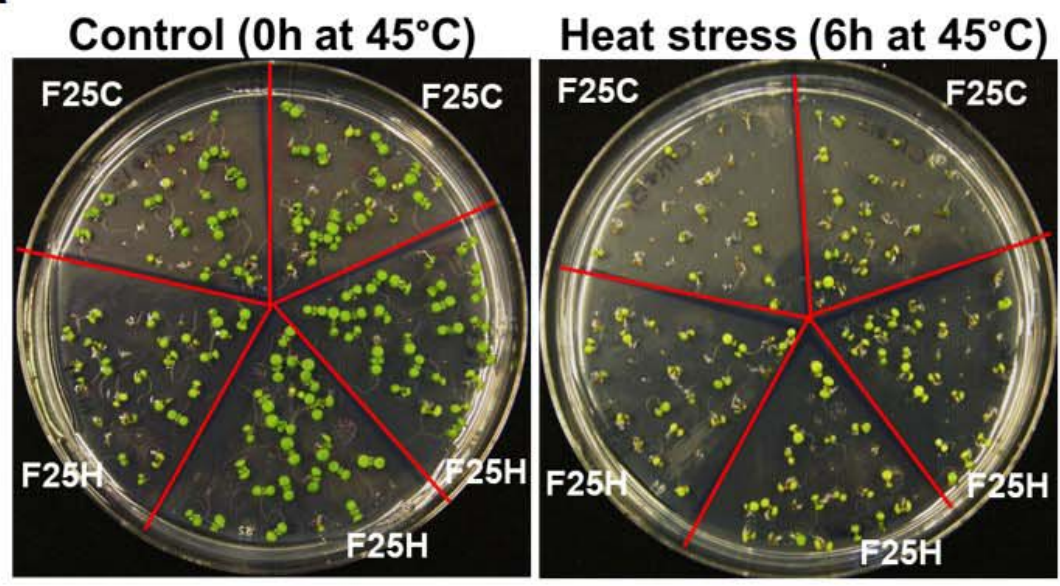

B

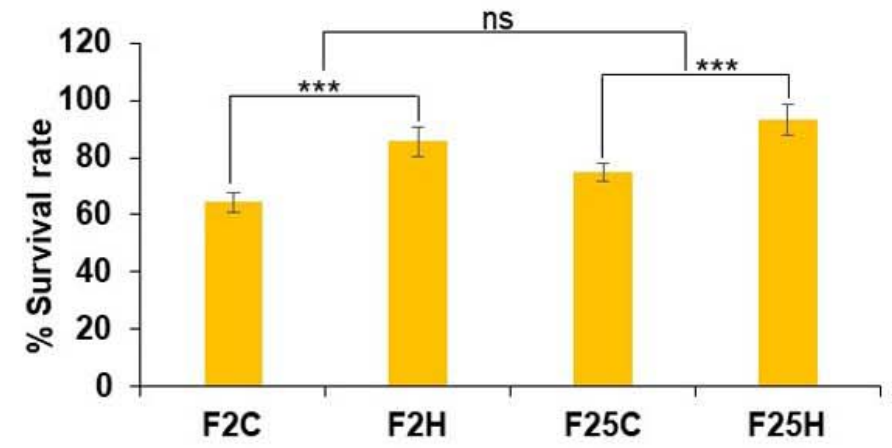

C a Incomplete copies of Luciferase
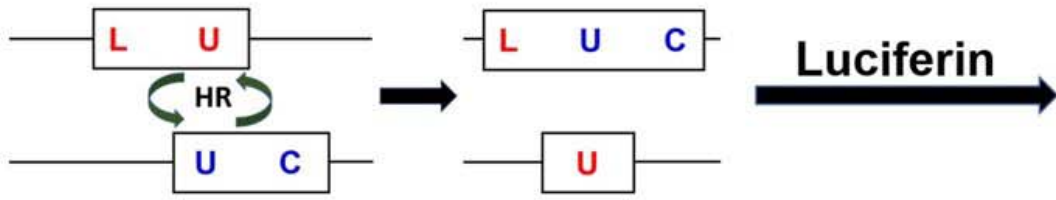

b $\quad$ F2C

F2H

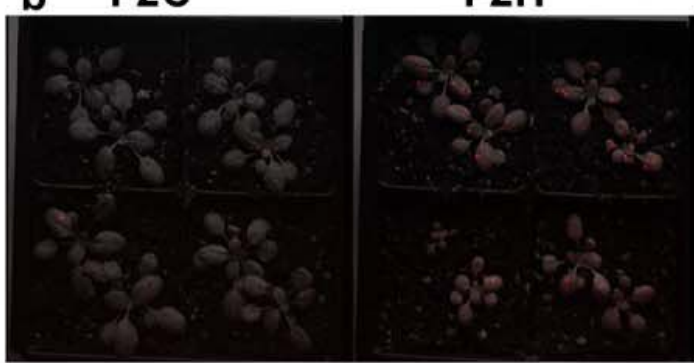

c $\mathrm{F} 25 \mathrm{C}$

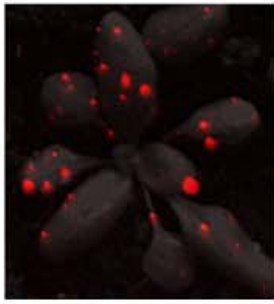

F25H

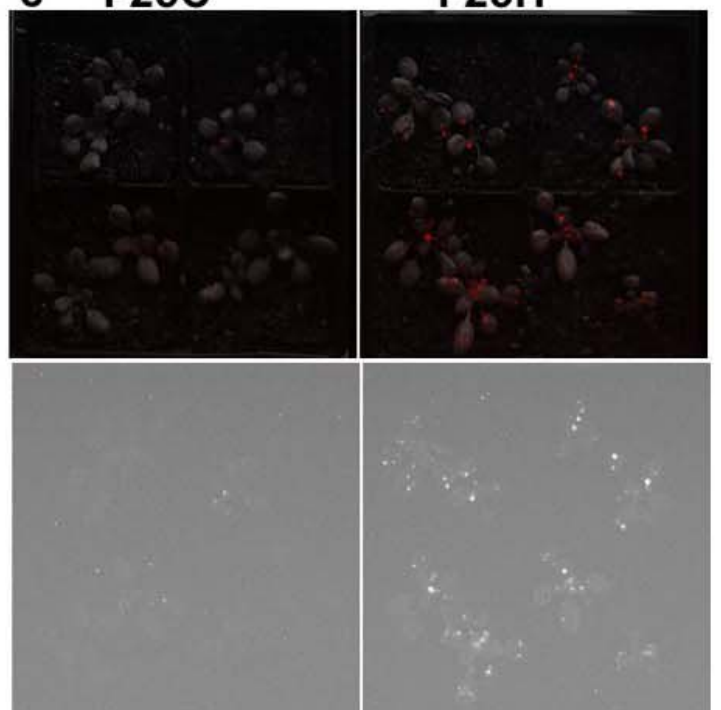


Figure 1. The heat-stressed progenies exhibited an increase in heat- stress resilience and higher recombination events. (A) Trends in the germination of the $\mathrm{F} 25 \mathrm{H}$ progeny under heat stress. Representative photographs of germination under control (0h) and heat-stress $(6 \mathrm{~h})$ conditions (the left panel). Seeds were either from the parallel control (F25C) or the heatstressed progeny $(\mathrm{F} 25 \mathrm{H})$. Each plate represents one set, and in total, five sets were used for each treatment. The right panel: The germination index graph. $0 \mathrm{~h}$ : non-stress control ( 0 hour at $45^{\circ} \mathrm{C}$ ); 6h: heat-stress ( 6 hours at $45^{\circ} \mathrm{C}$ ). Heat-stress was applied at $45^{\circ} \mathrm{C}$ for $6 \mathrm{~h}$, just 4 -days after stratification at $4^{\circ} \mathrm{C}$. The germination index was measured on the $6^{\text {th }}$ day. The data were analysed by two-way ANOVA with the Tukey's multiple comparisons test using GraphPad Prism version 8.4.2 for Windows. The data are shown as an average mean $\pm \mathrm{SD}$. A p-values less than 0.05 were considered statistically significant. Mean values that were significantly different from each other are indicated by $*(p<0.05), * *(p<0.01)$ and those that were nonsignificant (ns) is indicated by ns $(p>0.05)$. (B) Survival of mature plants of stressed progenies (F2H vs F2C and F25H vs F25C) under heat-stress. Heat- stress was given just before bolting for $96 \mathrm{~h}$ at $40^{\circ} \mathrm{C}$. A survival rate was calculated after 10-days of recovery and shown as an average percentage (with $\pm \mathrm{SD}$ ) of plants surviving the stress from three independent experiments. The data were analysed by one-way ANOVA with Tukey's multiple comparisons test using GraphPad Prism version 8.4.2 for Windows. The data are shown as an average percentage (with $\pm \mathrm{SD}$ ) of plants surviving the stress from three independent experiments. All $p$-values less than 0.05 were considered statistically significant. The asterisk shows a significant difference between the stressed progeny and parallel control progeny $(* * * p<0.001$, ns $p>0.05$ ). (C) Somatic HRF under non-stress conditions. Progenies of stressed plants exhibited a higher somatic homologous recombination (HRF) without exposure to heat-stress. (a) The scheme of the HRF analysis. Plant cells of the transgenic Arabidopsis thaliana line \#15d8 contain two overlapping copies of the disrupted non-functional luciferase gene. In cells where the transgene functionality is restored due to the event of homologous recombination, the luciferase activity can be detected as bright sectors after exposure to enzymatic substrate luciferine. (b) Recombination in F2C and F2H plants. (c) Recombination in F25C and F25H plants. In figures b and c, the upper panel is a superimposed photograph of HR events and plants, and the lower panel is an original florescence photograph. A minimum 50 plants per line were used in one independent experiment.

\section{The progeny of stressed plants exhibited a higher homologous recombination frequency (HRF)}

The F2H and F25H plants were analysed for HRF under non-stress conditions. The scheme of the HRF analysis is shown in Figure $1 \mathrm{C}(\mathrm{a})$. Both $\mathrm{F} 2 \mathrm{H}$ and $\mathrm{F} 25 \mathrm{H}$ stressed progenies exhibited a higher HRF as compared with their respective parallel controls (Figure 1C (b, c)). 


\section{Genetic and epigenetic changes in F2 and F25 progeny plants}

Genetic changes in F2C (the parental control), F25H (the stressed progeny) and F25C (the parallel control) were investigated by whole genome sequencing (WGS). The single nucleotide polymorphism (SNPs) and insertion/deletion (INDELs) were identified to explore the genetic diversity between the stressed $(\mathrm{F} 25 \mathrm{H})$ and the control progenies ( $\mathrm{F} 2 \mathrm{C}$ and $\mathrm{F} 25 \mathrm{C})$. Epigenetic changes were examined as an extent of changes in DNA methylation patterns using high-throughput bisulfite sequencing of the whole genome (WGBS). The distribution of epimutations induced by multigenerational heat-stress investigated in this study included differentially methylated positions (DMPs) and differentially methylated regions (DMRs). In total, fifteen genomes and fifteen methylomes were analysed which included five biological replicates of F2C, F25C, and F25H each.

\section{The multigenerational heat-stressed progeny exhibited higher SNPs and INDELs}

Genomic variants consisting of single nucleotide polymorphisms (SNPs) and insertions/deletions (INDELs) were explored. Their corresponding genomic locations such as the intronic, exonic, untranslated regions (5' UTR or 3' UTR), upstream and downstream of gene regions, and the intergenic regions were identified. The effects of these changes in coding sequences leading to synonymous or non-synonymous mutations were predicted. According to the SnpEff program (Cingolani et al. 2012) utilized in this study, upstream was defined as within $5 \mathrm{~kb}$ upstream of the distal transcription start site, and downstream was defined as $5 \mathrm{~kb}$ downstream of the most distal polyA addition site. Variants affecting the noncoding regions were expounded, and biotypes were identified with the available information after comparing with the TAIR 10 reference Arabidopsis genome.

Variant rates for the $\mathrm{F} 25 \mathrm{H}$ plants were 1 variant for every 1,700 bases, for F25C -1 variant for every $\sim 5,000$ bases, and for F2C -also 1 variant for every $\sim 5,000$ bases, suggesting that $\mathrm{F} 25 \mathrm{H}$ had $\mathrm{a} \sim 3 \mathrm{x}$ higher rate of variance in its genome when all samples were jointly considered (Supplementary Information). The total SNPs identified for F25H, F2C and F25C were 53,678, 15,599, 15,526, respectively (Figure 2A). The total numbers of INDELs identified for F25H, F2C and F25C were 17,669, 7,986, 8,143, respectively (Figure 2A). After combining SNPs and INDELs, the total genetic variations in F25H, F2C and F25C were 71,347, 23,585, and 23,669, respectively (Figure 2A). The parallel F25C and the parental F2C controls exhibited a similar range with a slight difference between their 
numbers of SNPs and INDELs (Figure 2A). In summary, the F25H heat-stressed progeny showed over three times more genetic variations than the parental and parallel controls, suggesting that heat-stress induced genetic variations in the stressed progeny (Figure 2A).

Interestingly, INDELs were of a similar size in F25C and $\mathrm{F} 2 \mathrm{C}$, whereas they were significantly larger in $\mathrm{F} 25 \mathrm{H}$ (Figure 2B), suggesting that multigenerational heat-stress resulted in an increase in the size of INDELs in the stressed progeny.

The GATK Haplotype caller calls SNPs and INDELs via an assembly of haplotypes in the active region of the genome which enables the identification of variants that are unique to each sample genome by extracting the non-overlapping sites specific for $\mathrm{F} 25 \mathrm{H}, \mathrm{F} 25 \mathrm{C}$, and F2C. The analysis of non-overlapping site-specific variants revealed 39,712, 1,313 and 1,308 unique SNPs for the F25H, F2C, and F25C plants, respectively; 11,478, 1,576 and 1,801 unique INDELs were identified for F25H, F2C and F25C, respectively (Figure 2C). Thus, the F25H plants showed a dramatically higher number of unique SNPs and INDELS than the control plants; specifically, there was over a 30-fold higher number of SNPs and over a 6-fold higher number of INDELS in F25H as compared with either control group, suggesting that exposure to multigenerational heat predominantly led to the generation of SNPs (Figure 2C). The parental F2C and parallel F25C controls showed a very similar number of unique SNPs (Figure 2C). 


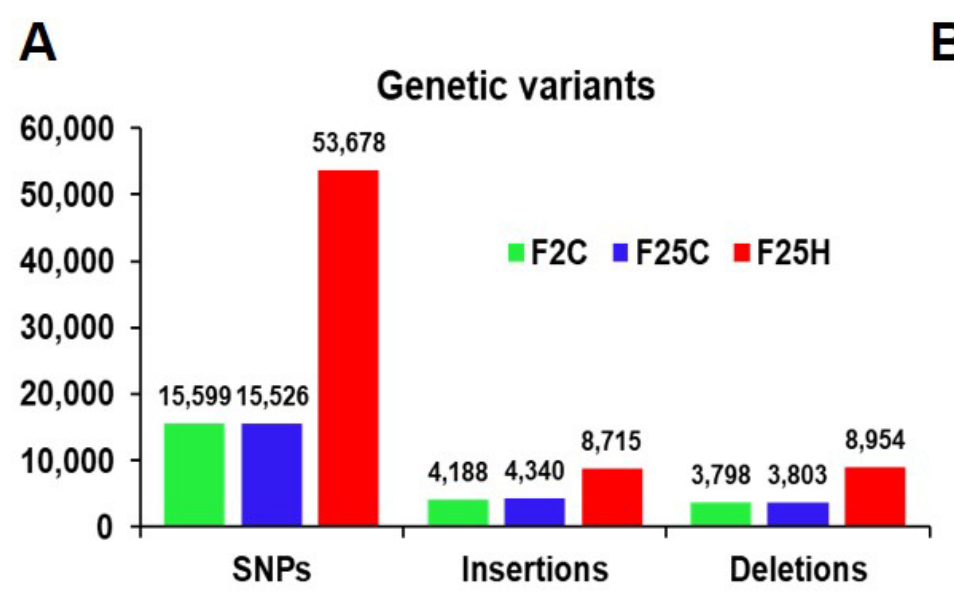

C

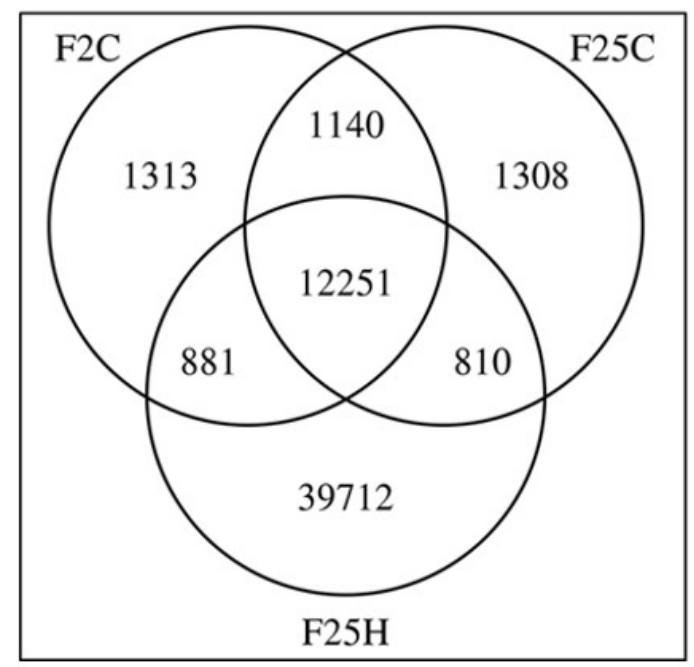

B

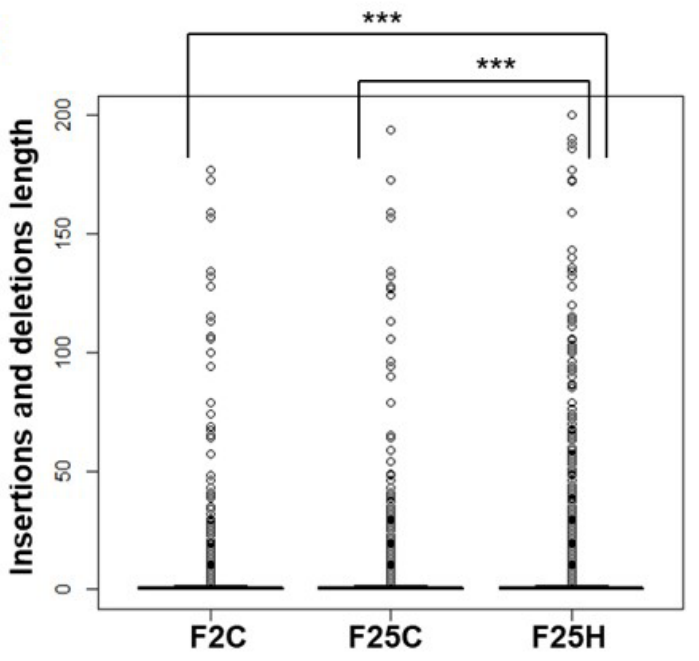

SNPS

INDELS

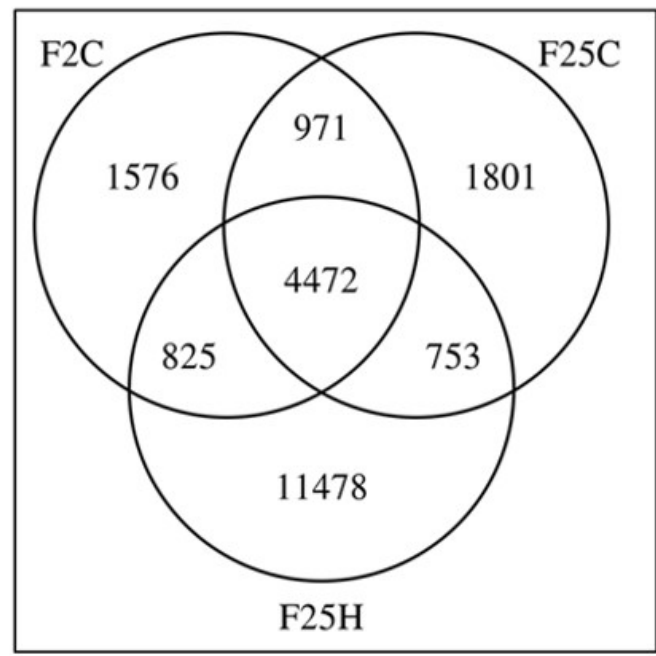

D

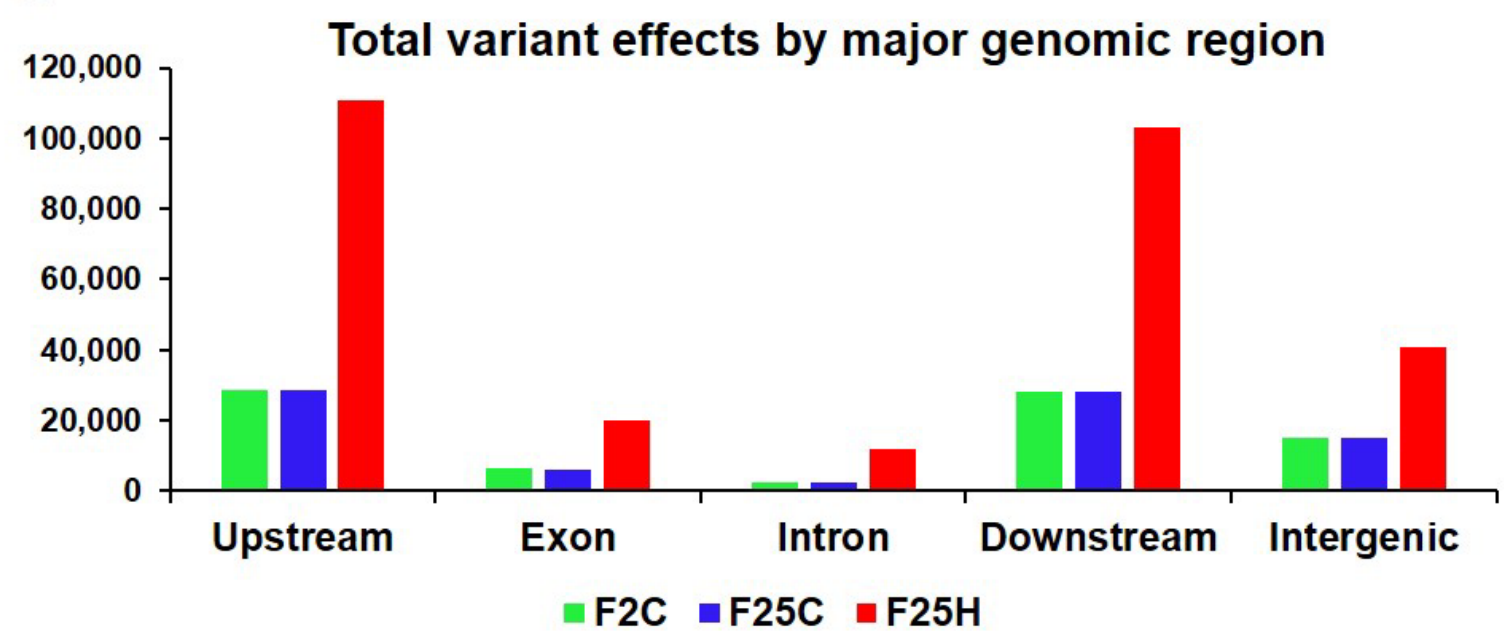


Figure 2. The heat-stressed progeny showed a higher number of genetic variations. (A) The total number of SNPs and INDELs in the genomes of stressed progeny and in the parallel and parental control progenies when all samples were jointly considered. (B) The box plot for the length of insertions and deletions of variants within F2C, F25C and F25H. The asterisk above bracket (*) shows a significant difference between F25H vs. F2C and F25H vs. F25C where $* * *$ indicates $p<0.001$. Wilcoxon rank-sum text was used to determine a statistical difference $(p<0.05)$. (C) A Venn diagram of jointly called variants. The non-overlapping area of Venn indicates the number of unique SNPs and INDELs in each sample group. Sample variants were jointly called by the "GATK haplotype Caller" using five samples together. (D) The total number of variant effects by major genomic regions. The number of variant effects in the major genomic regions are shown above each bar, and the genomic regions are indicated on the x-axis. According to the SnpEff program (Cingolani et al. 2012) utilized in this study, upstream was defined as within $5 \mathrm{~kb}$ upstream of the distal transcription start site, and downstream was defined as $5 \mathrm{~kb}$ downstream of the most distal polyA addition site. Variants affecting the non-coding regions were expounded, and biotypes were identified with the available information after comparing with the TAIR 10 reference Arabidopsis genome.

\section{The multigenerational heat-stressed progeny exhibited a higher number of genetic variant effects}

The total number of genetic variant effects calculated by the corresponding genomic locations of SNPs and INDELs such as the intronic, exonic, untranslated regions (5' UTR or 3'UTR), upstream and downstream of gene regions and intergenic regions were identified, and coding effects were predicted in respect to protein-coding genes such as synonymous or nonsynonymous mutations. Positional profiles of genetic variations (SNPs and INDELs) revealed a higher number of variant effects in the $\mathrm{F} 25 \mathrm{H}$ heat-stressed progeny compared with the nonstressed parallel F25C and parental F2C progenies (Figure 2D). The F25H plants showed the highest total number of variant effects $(2,93,405)$ compared with the F25C $(82,179)$ and F2C $(81,657)$ controls (Figure 3). The classification of variant effects by genomic regions revealed the largest number of effects observed in upstream of $(\mathrm{F} 25 \mathrm{H}-110,837$; F25C - 28,762; F2C 28,403) followed by downstream (F25H - 103,301; F25C - 28,035; F2C - 27,947) and intergenic regions (F25H - 40,941; F25C - 15,184; F2C - 15,058) (Figure 2D, 3). Variant effects were also found within the 5' untranslated (F25H - 1,817; F25C - 438; F2C - 442) and the 3' untranslated regions (F25H - 2,312; F25C - 461; F2C - 442) of the genome (Figure 3). 
Other regions that also showed some variants are represented in Figure 3. The F25H plants showed the highest number of synonymous mutations (that do not alter the amino acid sequence; 3,891) and nonsynonymous mutations (that alter the amino acid sequence; 4,510) than the F25C plants (313 - synonymous, 752 - non-synonymous) and the F2C plant controls (316 - synonymous, 742 - non-synonymous) (Figure 3). Overall, the F25H heatstressed progeny showed a higher number of 12x and 6x? synonymous and nonsynonymous mutations, respectively, than the parallel and parental control progenies. In F25H, there were identified 18 SNP missense mutations converting a stop codon into an amino acid coding triplet and 66 SNP nonsense mutations generating a stop codon sequence (Figure 3C). These numbers were much lower in F25C and F2C where 5 and 5 stop codon losses and 17 and 10 stop codon gains were observed, respectively (Figure 3A, B). The non-synonymous mutations result in a biological variation in the living organism, consequently, they are subjected to natural selection. 
bioRxiv preprint doi: https://doi org/10.1101/20201130.405365. this version posted December 22020 . The copyright holder for this preprint (which was not certified by peer review) is the author/funder, who has granted bioRxiv a license to display the preprint in perpetuity. It is made available under aCC-BY 4.0 International license.

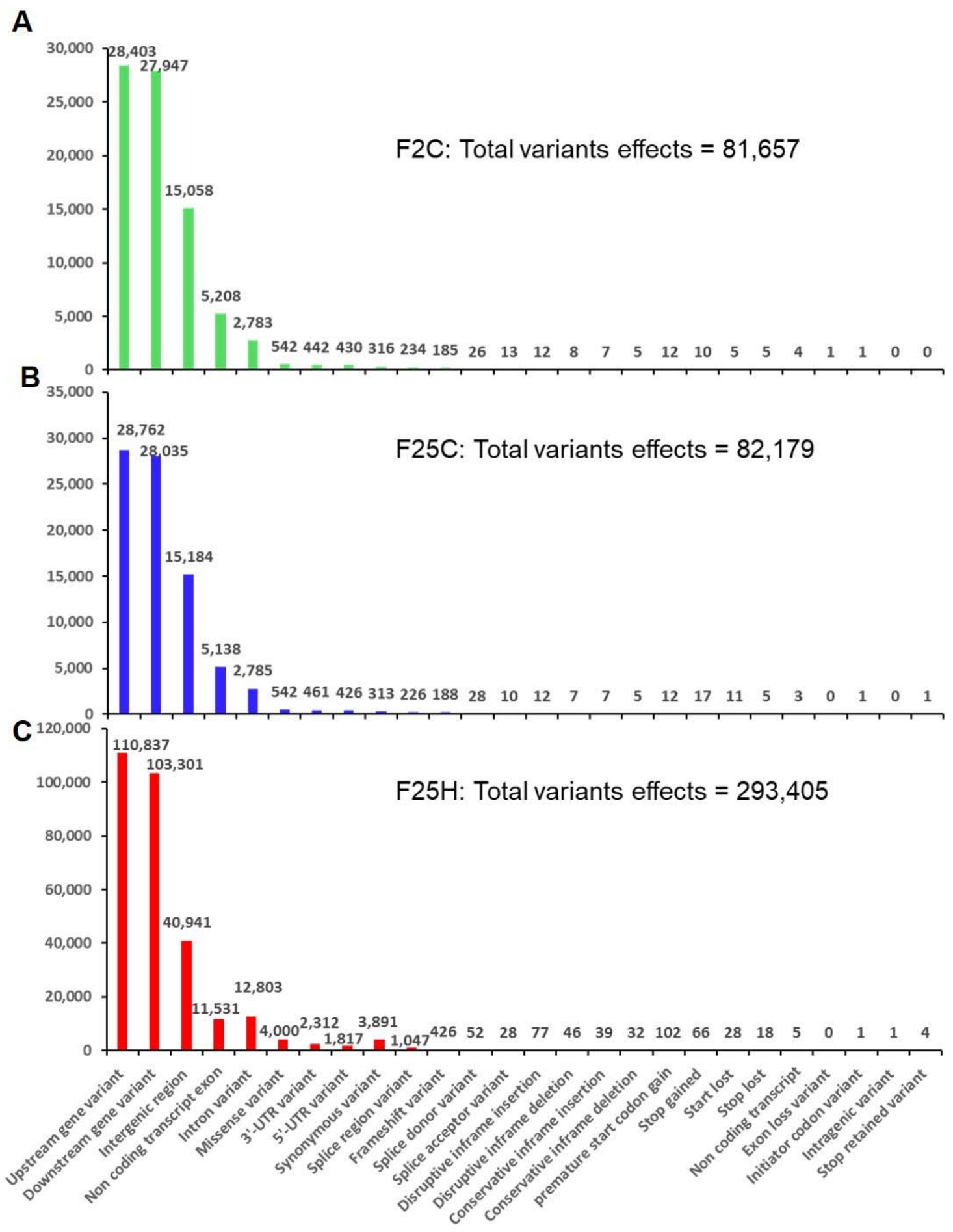


Figure 3. The distribution of variants across the genome by their potential effects based on genomic positions. (A) F25H, (B) F25C and (C) F2C. The number of variant effects are shown above each bar, and effects from SnpEff are listed on the X-axis. According to the SnpEff program (Cingolani et al. 2012) used in this study, upstream was defined within $5 \mathrm{~kb}$ upstream of the distal transcription start site, and downstream was defined as $5 \mathrm{~kb}$ downstream of the most distal polyA addition site. The variants affecting non-coding regions were expounded, and biotypes were identified with available information after comparing with the TAIR 10 version of the reference Arabidopsis genome.

\section{The analysis of epigenetic variations}

\section{Multigenerational exposure to heat stress reduces the percentage of total global DNA methylation in the $\mathrm{CHG}$ and $\mathrm{CHH}$ contexts}

The sequence data mapped to the reference Arabidopsis genome revealed an average of 16,081,856 methylated cytosine residues (mCs), 16,095,139 $\mathrm{mCs}$ and 15,869,955 $\mathrm{mCs}$ for F2C, F25C, and F25H respectively, representing all three-sequence context. The bisulfite sequencing data revealed that the average percentage of global genome methylation in the $\mathrm{CG}$ context did not show any significant difference between the $\mathrm{F} 25 \mathrm{H}$ plants and either of the control samples (Figure 4A). However, in the CHG context, the F25H plants showed the lower global methylation levels $(p<0.10)$ than the controls (Figure 4A). Similar data were found for in the $\mathrm{CHH}$ context, the $\mathrm{F} 25 \mathrm{H}$ plants exhibited a significantly lower methylation $(p<0.05)$ compared with the controls (Figure 4A). The reduction of methylation levels in the $\mathrm{CHG}$ and $\mathrm{CHH}$ contexts might be a part of adaptation strategies to heat stress.

\section{The analysis of the number of DMPs and DMRs reveals a higher number of differentially} methylated cytosines in the $\mathrm{CHG}$ and $\mathrm{CHH}$ contexts in the F25H plants

DNA methylation changes (epimutations) between the F25H vs. F2C and F25H vs. F25C comparison groups were considered to be potentially induced by multigenerational exposure to heat stress, and epimutations between $\mathrm{F} 25 \mathrm{C}$ vs. F2C were considered as spontaneously/stochastically induced differences in the epigenome of the parental and advanced control progenies. The total number of DMPs for F25H vs. F2C, F25H vs. F25C and F25C vs. F2C were 66,491, 78,412 and 80,464, respectively (Figure 4B). The analysis of DMPs in a specific sequence context revealed a different pattern. While DMPs in the CG context were higher in the $\mathrm{F} 25 \mathrm{C}$ vs. F2C control comparison group in the $\mathrm{CHG}$ and $\mathrm{CHH}$ contexts, DMPs were much higher in comparison groups involving the $\mathrm{F} 25 \mathrm{H}$ stressed progeny (Figure 4C). The difference was especially obvious for hypomethylated cytosines at the CHG context; the F25H vs. F2C group showed 299 hypomethylated DMPs, while the 
F25C vs. F2C group - only 230. A similar pattern was observed in the CHH context, nearly a 2-fold larger number of hypomethylated DMPs was observed in the F25H vs. F2C group in comparison with the F25C vs. F2C group - 342 vs. 176, respectively; the difference for hypermethylated DMPs was also substantial, 133 vs. 87 (Figure 4C). These data suggest that epimutations induced by multigenerational heat stress are primarily associated with changes in the $\mathrm{CHG}$ and $\mathrm{CHH}$ contexts, and that hypomethylation is likely a prevalent mechanism.

The total DMRs in the-100 bp window for F25H vs. F2C, F25H vs. F25C and F25C vs. F2C were 10,314, 12,940, 12,619, respectively (Figure 5B). In the case of the 1000-bp window, the total DMRs in for F25H vs. F2C, F25H vs. F25C and F25C vs. F2C were 49, 36, 32, respectively. As to DMPs in the $\mathrm{CHG}$ and $\mathrm{CHH}$ context, the total number of DMRs in the 1000-bp window revealed the larger number of differentially methylated DMRs in the F25H vs. F2C group compared with other groups (Figure 5C).

The analysis of differential methylation events in the genomic context reveals no difference between test groups

The biological impact of all differential methylation events observed was put into the genomic context with the subsequent analysis detailing variable regions and positions of methylation within the gene structure and sequence islands. To obtain insights into the relationship of DMPs with the promoter regions, we calculated their distance to the nearest transcription start site (TSS).

The distribution collected by the absolute distance of individual DMPs in sample replicates revealed that the differentially methylated DMPs in the CG context were located approximately within 0 to $2 \mathrm{~kb}$ to TSS (Figure 4D). The overall nucleotide distance to TSS dropped for DMPs in the $\mathrm{CHG}$ and $\mathrm{CHH}$ contexts ranging from 0 to $1 \mathrm{~kb}$ (Figure 4D). A similar picture was observed for DMRs (Figure 5A). Curiously, the larger range of distances to TSS was observed in the comparison groups involving $\mathrm{F} 25 \mathrm{H}$. 

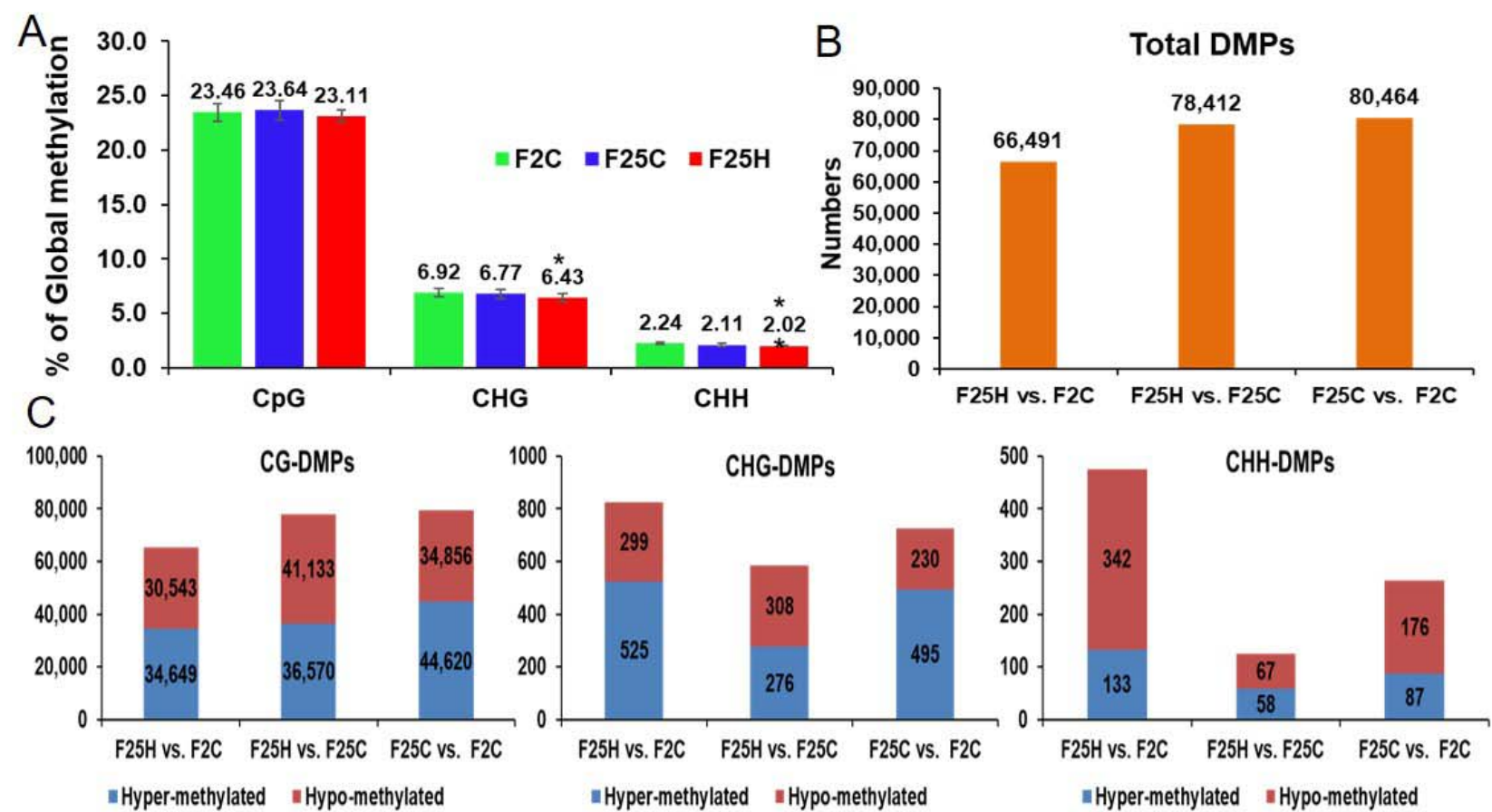

"Hyper-methylated $\quad$ Hypo-methylated
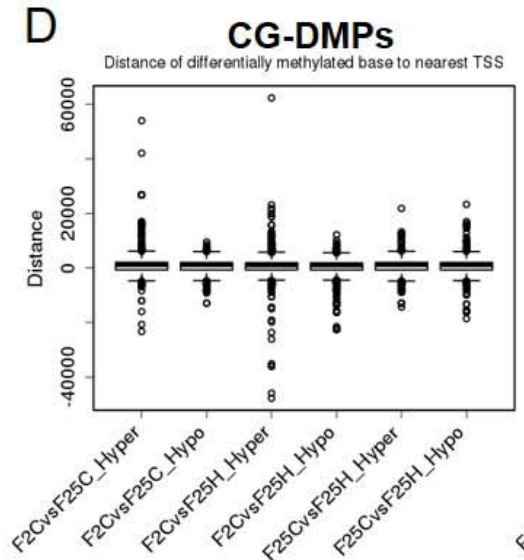

CHG-DMPs

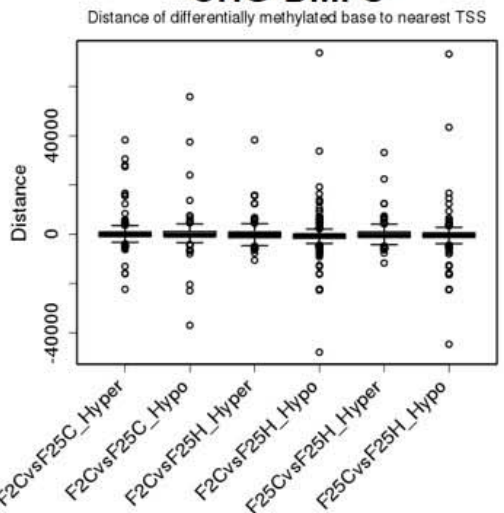

$\mathrm{E}$

\section{CpG methylation clustering}

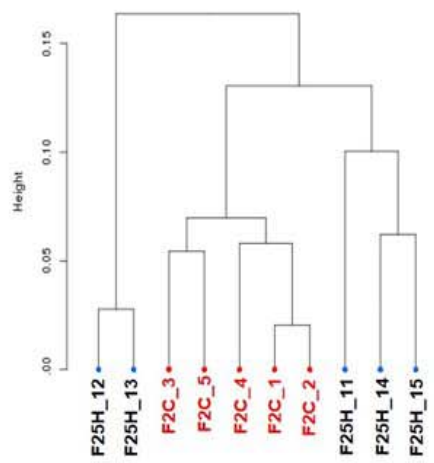

CHG methylation clustering

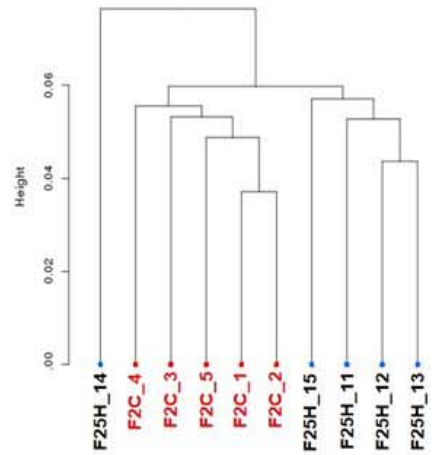

\section{CHH-DMPs}

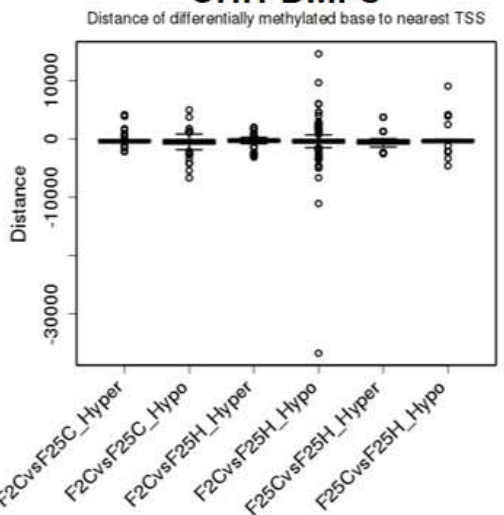

\section{$\mathrm{CHH}$ methylation clustering}

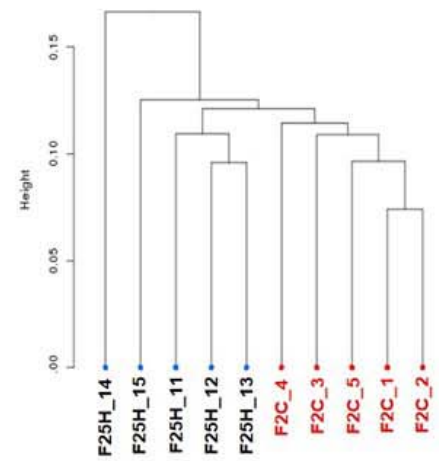


Figure 4. Global methylation changes and differentially methylated positions (DMPs). (A) The average percentage of methylated cytosines in $\mathrm{F} 2 \mathrm{C}, \mathrm{F} 25 \mathrm{C}$, and $\mathrm{F} 25 \mathrm{H}$ in the CG, $\mathrm{CHG}$, and $\mathrm{CHH}$ sequence contexts $(\mathrm{H}=\mathrm{A}, \mathrm{T}, \mathrm{C}$ and mean, $\mathrm{n}=5)$. Methylation levels were determined from reads with the minimum coverage $\geq 10$ mapped to the TAIR 10 reference genome by using Bismark software. The data were analysed by one-way ANOVA with the Tukey's multiple comparisons test using GraphPad Prism version 8.4.2 for Windows. The data are shown as the average percentage (with $\pm \mathrm{SD}$ ) of methylated cytosines from five individual methylomes in each of the progenies. The asterisk shows a significant difference between the stressed progeny and the parental control progeny $(* p<0.1, * * p>0.05)$. (B) The total number of DMPs in F25H vs. F2C, F25H vs. F25C and F25C vs F2C. (C) The total number of DMPs in the $\mathrm{CG}, \mathrm{CHG}$ and $\mathrm{CHH}$ contexts in $\mathrm{F} 25 \mathrm{H}$ vs. F2C, F25H vs. F25C and F25C vs F2C. (D) The distance of DMPs to the nearest TSS in the CG context overlapping by $>50 \%$, in the $\mathrm{CHG}$ context overlapping by $>25 \%$ and in the $\mathrm{CHH}$ context overlapping by $>25 \%$. The distance to TSS for differentially methylated $\mathrm{CG}, \mathrm{CHG}$ and $\mathrm{CHH}$ are plotted by the hyper/hypomethylation analysis of F25H vs. F2C, F25C vs. F2C, and F25H vs. F25C. The Y-axis shows the nucleotide distance to a transcription start site. (E) Global methylation clustering of DMPs in the $\mathrm{CG}, \mathrm{CHG}$ and $\mathrm{CHH}$ contexts. Hierarchical clustering of $\mathrm{F} 25 \mathrm{H}$ vs. F2C using 1-Pearson's correlation distance.

The progeny exposed to multigenerational heat-stressed did not show any difference in the

\section{frequency distribution of methylation}

The percentage of frequency distribution of methylation (the analysis of the percentage of methylated cytosines at specific sites) in $\mathrm{F} 2 \mathrm{C}, \mathrm{F} 25 \mathrm{C}$, and $\mathrm{F} 25 \mathrm{H}$ showed that most of the cytosines had either a high $(70-100 \%)$ or a low (0-10\%) level of methylation in the methylated CG context, however we did not see any difference in frequency distribution between F2C, F25C, and F25H progeny epigenomes (Supp. Figure S1). Cytosine methylation in the CHG context was much lower in frequency compared with the CG context; most sites had $0-60 \%$ of the methylation level including very high frequency at $0-10 \%$, and sites with high levels of methylation (70-100\%) were not observed (Supp. Figure S2). Similarly, methylation in the $\mathrm{CHH}$ context was much lower than in the $\mathrm{CG}$ or CHG contexts; there was an even low frequency of occurrence of sites with the 0-20\% methylation level (Supp. Figure S3). When each sequence context was considered, there was no significant difference in the frequency of methylation levels between the $\mathrm{F} 2 \mathrm{C}, \mathrm{F} 25 \mathrm{C}$, and $\mathrm{F} 25 \mathrm{H}$ groups. In general, F2C, $\mathrm{F} 25 \mathrm{C}$, and $\mathrm{F} 25 \mathrm{H}$ showed a similar percentage of distribution patterns of methylation in the CG, CHG, and CHH contexts (Supp. Figure S1-3). 


\section{Hierarchical clustering analysis identified directional changes of heat-induced epimutations in the stressed progeny}

Hierarchical clustering analysis between samples of the parental control (F2C) and stressed $(\mathrm{F} 25 \mathrm{H})$ progenies showed that all parental individuals siblings (F2C) clustered together in all cytosine methylation contexts. However, in the case of the F25H stressed progeny in the CG context, three out of five individuals clustered together, and the other two also showed more closeness (on the basis of branch height) compared with F2C (Figure 4E). In the CHG context, four out of five individuals clustered together, and the other one also showed more closeness compared with F2C (Figure 4E), which possibly suggests the directional changes of heat-induced epimutations. Interestingly, in the $\mathrm{CHH}$ context, there was a complete separation between the $\mathrm{F} 2 \mathrm{C}$ and $\mathrm{F} 25 \mathrm{H}$ groups (Figure 4E). Considering that $\mathrm{CHH}$ methylation happens in plants de novo, the clustering together of all stressed individuals suggests the importance of $\mathrm{CHH}$ methylation in stress adaptation and indicates that multigenerational exposure to heat stress likely induces non-random changes in the methylome in the $\mathrm{CHH}$ context.

To further determine whether the effects of multigenerational exposure to heat stress on epimutations were directional, we analysed clustering of $\mathrm{F} 2 \mathrm{C}$ and $\mathrm{F} 25 \mathrm{H}$ plants with $\mathrm{F} 25 \mathrm{C}$ plants. The analysis of DMPs in the CG context showed that most of the non-stressed parallel control plants $(\mathrm{F} 25 \mathrm{C})$ clustered with the parental generation plants $(\mathrm{F} 2 \mathrm{C})$, whereas individuals of the stressed progeny segregated as an independent cluster of parental plants (Supp. Figure S4-7). The pairwise comparisons and Principal Component Analysis (PCA) plots (Supp. Figure S4A) derived by the analysis of similarities in global methylation levels at the CG sites confirmed the outlined similarities, further confirming the homogeneity of all tested samples. Hierarchical clustering of methylation profiles at CHG (Supp. Figure S4B, S6) and $\mathrm{CHH}$ (Supp. Figure S4C, S7) sites displayed similar clusters of sample groupings as in their CG profile, with $\mathrm{F} 25 \mathrm{C}$ and $\mathrm{F} 2 \mathrm{C}$ being mainly clustered together. These results suggest that the effects of multigenerational exposure to heat stress on epimutations is directional, due to the fact that when individuals of the $\mathrm{F} 2 \mathrm{C}$ parental control progeny and the F25C parallel control progeny cluster together, they exhibit more similar DNA methylation patterns (Supp. Figure S4-7). This was also the case of DMRs in CG (Supp. Figure S8A), CHG (Supp. Figure S8B) and CHH (Supp. Figure S8C) contexts.

In summary, hierarchical clustering of samples based on DNA methylation patterns of differentially methylated positions (DMPs) (Figure 4E, Supp. Figure S4-S7) and differentially methylated regions (DMRs) (Supp. Figure S8) separates the stressed plants and 
bioRxiv preprint doi: https://doi.org/10.1101/2020 11.30.405365; this version posted December 2, 2020. The copyright holder for this preprint

(which was not certified by peer review) is the author/funder, who has granted bioRxiv a license to display the preprint in perpetuity. It is made available under aCC-BY 4.0 International license.

non-stressed plants into distinct groups, suggesting that multigenerational exposure to heat stress impacts DNA methylation patterns in a directional way to shape the epigenome of stressed progeny. 
bioRxiv preprint doi: https://doi.org/10.1101/2020.11.30.405365; this version posted December 2, 2020. The copyright holder for this preprint (which was not certified by peer review) is the author/funder, who has granted bioRxiv a license to display the preprint in perpetuity. It is made available under aCC-BY 4.0 International license.

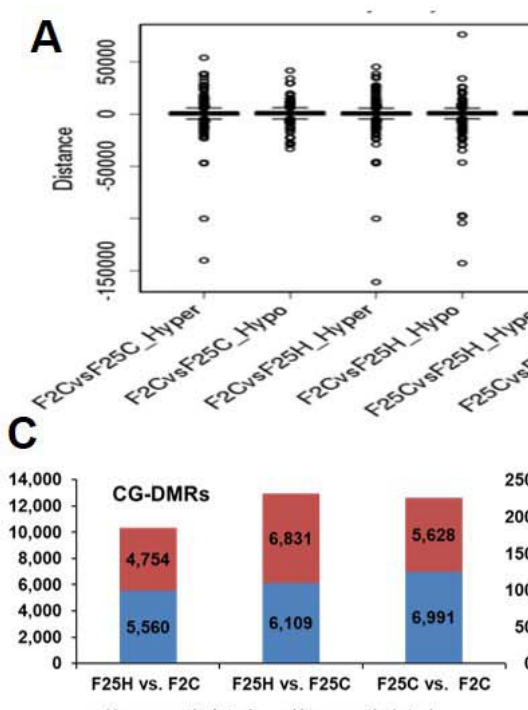

- Hyper-methylated $=$ Hypo-methylated

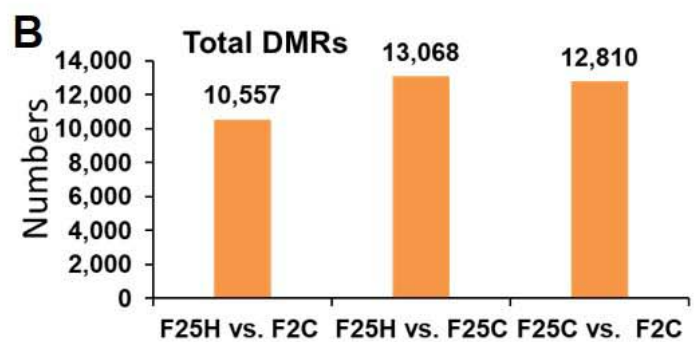

F25H vS. F2C F25H vs. F25C F25C vs. F2C
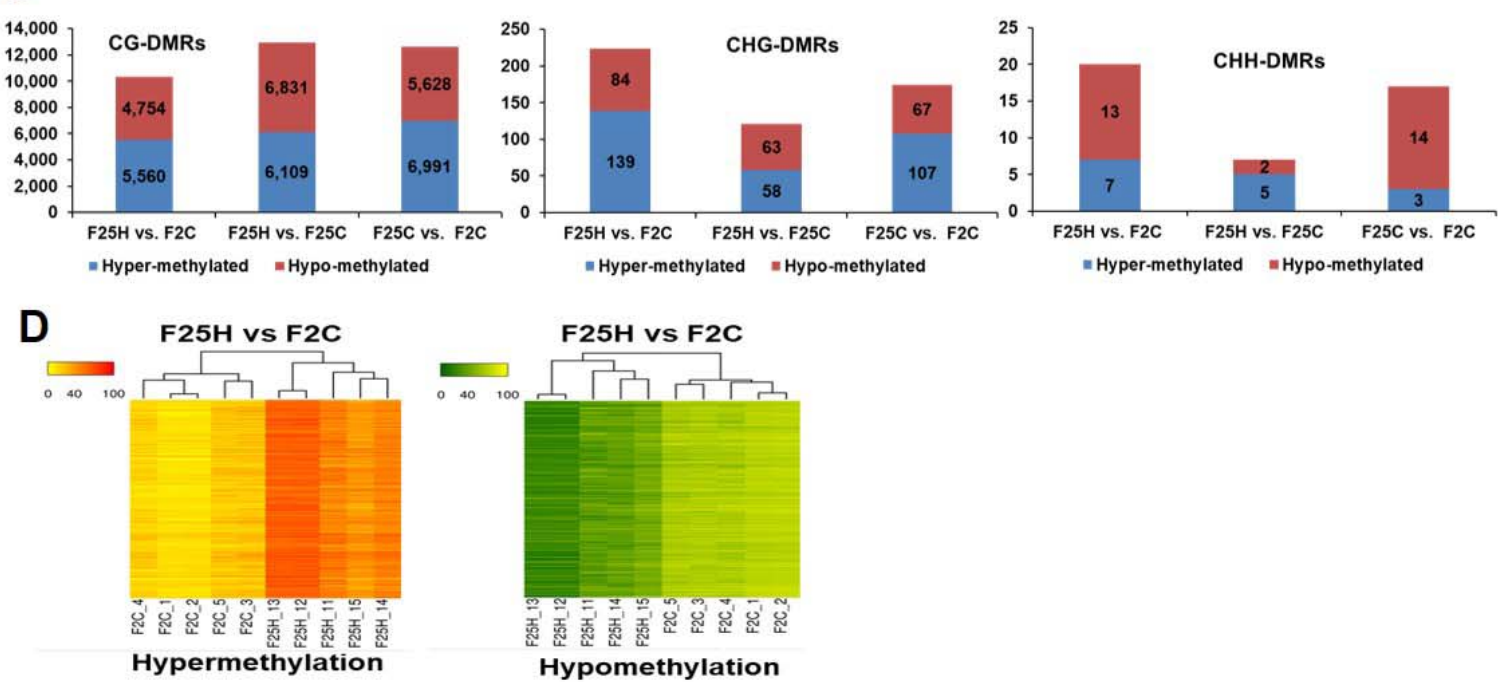

E
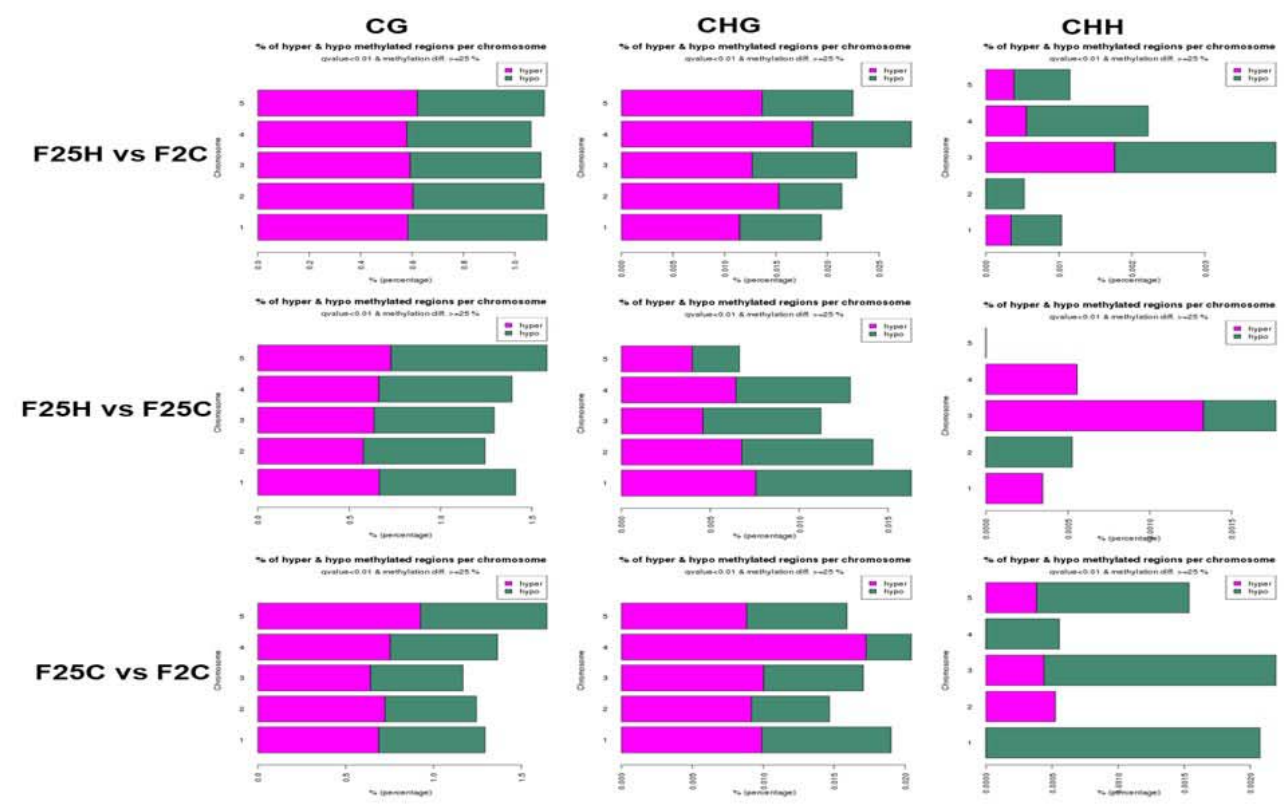
Figure 5. Differentially methylated regions (DMRs). (A) Differentially methylated regions (DMRs) overlapping by $>25 \%$. The distance to TSS in differentially methylated regions are plotted by $\mathrm{F} 25 \mathrm{H}$ vs. F2C, F25C vs. F2C and F25H vs. F25C hyper/hypomethylation comparisons. The Y-axis shows the nucleotide distance to a transcription start site. (B) The total number of DMRs in F25H vs. F2C, F25H vs. F25C and F25C vs F2C comparison groups. (C) The total number of DMRs in $\mathrm{CG}, \mathrm{CHG}$ and $\mathrm{CHH}$ contexts in F25H vs. F2C, F25H vs. F25C and F25C vs F2C. (D) Heat maps of DMRs of hypermethylated cytosines (the upper panel) and hypomethylated cytosines (the lower panel) in CG contexts in F25H vs. F2C. Differentially methylated positions in the genome with differences $>50 \%$ showing the percentage of methylation in $\mathrm{F} 25 \mathrm{H}$ vs. F2C. In the left panel, the red color indicates the larger percentage of methylation, and the yellow color indicates the lower percentage; while in the right panel, the green color indicates the larger percentage of methylation and the yellow one indicates the lower percentage, q-value $<0.01$. (E) The percentage of hyper- and hypomethylated DMR distributions through the chromosome level. DMRs per chromosome per sequence context over a 100- bp window in the CG context (the left panel), DMRs in the CHG context (the middle panel) and DMRs in the CHH context (the right panel). DMRs with differences $>25 \%$ show the percentage of hyper- and hypomethylated regions; the pink section indicates the percentage of hypermethylation, and green sections indicates the percentage of hypomethylation, q-value $<0.01$.

\section{The heatmaps based hierarchical clustering analysis of DMPs and DMRs revealed directional changes of heat-induced epimutations in the stressed progeny}

We analyzed the relatedness of the control and stressed progeny samples using the hierarchical clustering heatmap analysis (Figure 6A). The heatmap analysis of changes in both hypo- and hyper-methylated DMPs revealed that the F25H stressed progeny showed a clear separation of their five samples from five samples of the $\mathrm{F} 2 \mathrm{C}$ parental progeny in all three $\mathrm{CG}, \mathrm{CHG}$ and $\mathrm{CHH}$ cytosine methylation contexts (Figure 6A). Similarly, a comparison between F25H and F25C also showed separate clustering (Supp. Figure S9A). A comparison between F25C and F2C samples showed more similarity, with two F25C samples clustering together with F2C samples (Supp. Figure 9B), CG- Heatmap clustering of DMRs also exhibited a clear separation between F25H and F2C samples (Figure 5D). In heatmap analysis, the separation of $\mathrm{F} 25 \mathrm{H}$ individuals from F2C parental and F25C parallel control 
individuals likely indicates the directionality of epimutations in the stressed progeny due to multigenerational exposure to heat stress.

\section{The distribution of DMPs and DMRs through chromosomes and generational changes}

The percentage of hypermethylated and hypomethylated DMPs per chromosome in the CG context showed an almost equal proportion of hyper- and hypo-methylation across all five chromosomes (Figure 6B). Although the average number of methylated bases in the CHG and $\mathrm{CHH}$ profiles were slightly higher than those in $\mathrm{CG}$, the distribution of methylation levels varied across the different chromosomes in each sequence context. Hypermethylation and hypomethylation were apparent in the CG context (Figure 6B), while the CHG context (Figure 6B) had more hypermethylated bases distributed at the chromosome level in comparison groups of F25H vs F2C and F25C vs F2C. Hypomethylation was mostly distributed in the $\mathrm{CHH}$ context (Figure 6B) and less distributed in the CHG context in all comparison groups. F25C vs. F2C had more hypermethylated bases than hypo-methylated bases per chromosome in the $\mathrm{CHG}$ context. In the $\mathrm{CHH}$ context, more hypomethylated bases per chromosome were found in all compared samples, except chromosome three which had an almost equal distribution of hyper- and hypomethylated bases (except F25H vs F2C) and the higher percentage of methylation than other chromosomes (Figure 6B). The proportions of DMPs per chromosome observed in F25H vs. F2C revealed more of hyper-methylated DMPs in the CHG context (Figure 6B), while in the CHH context, there were more hypomethylated DMPs per chromosome (Figure 6B). The analysis of hyper/ hypo methylated events per chromosome indicates that $\mathrm{F} 25 \mathrm{H}$ has a significant proportion $(\mathrm{p}<0.01)$ of hypermethylation in the CHG context (Figure 6B) and hypo-methylation in the $\mathrm{CHH}$ context (Figure 6B) compared with the non-stressed parental generation F2C. Similarly, the nonstressed parallel progeny F25C also shows a significant proportion $(\mathrm{p}<0.01)$ of hypermethylation compared with the parental generation F2C in the CHG context (Figure 6B) and hypo-methylation in the $\mathrm{CHH}$ context (Figure 6B).

The differentially extracted methylated regions (DMRs) show the same trends to differentially methylated positions (DMPs) in both the hyper- and hypomethylated contexts. The distribution of methylated regions highlights more hypermethylation in the CHG context and slightly more hypermethylation in the CG context; it also shows hypomethylation at the CHH context when F25H vs. F2C are compared in a 100-bp window (Figure 5E). When the sliding window was increased to 1000_bp, the proportion of hypermethylated DMRs per 
bioRxiv preprint doi: https://doi.org/10.1101/2020 11.30.405365; this version posted December 2, 2020. The copyright holder for this preprint

(which was not certified by peer review) is the author/funder, who has granted bioRxiv a license to display the preprint in perpetuity. It is made available under aCC-BY 4.0 International license.

chromosome increased in CG and CHG contexts. DMRs in the CHH context in the 1000-bp sliding window showed no DMRs per chromosome (Supp. Figure S10). 
bioRxiv preprint doi: https://doi.org/10.1101/2020.11.30.405365; this version posted December 2, 2020. The copyright holder for this preprint (which was not certified by peer review) is the author/funder, who has granted bioRxiv a license to display the preprint in perpetuity. It is made available under aCC-BY 4.0 International license.

A
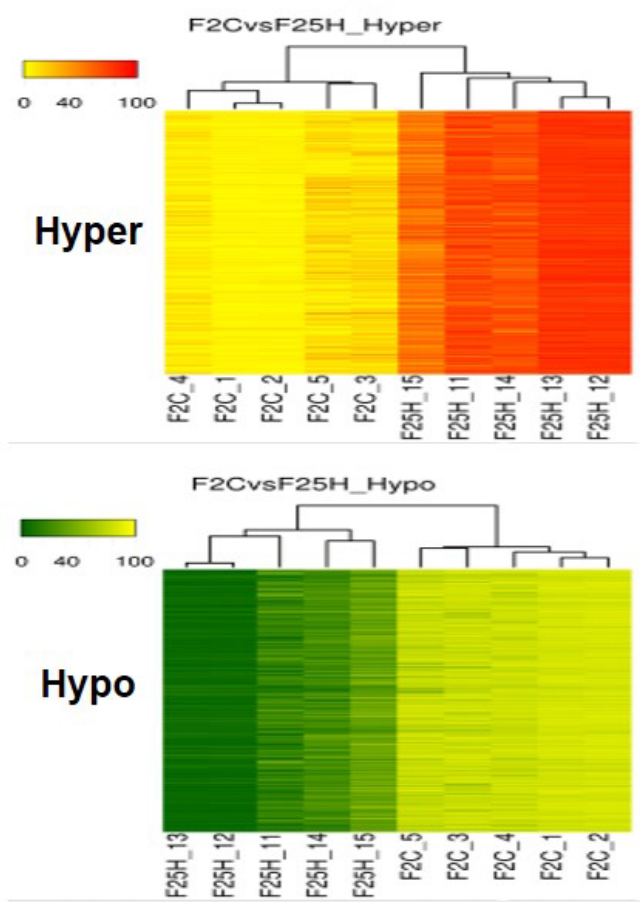

CHG
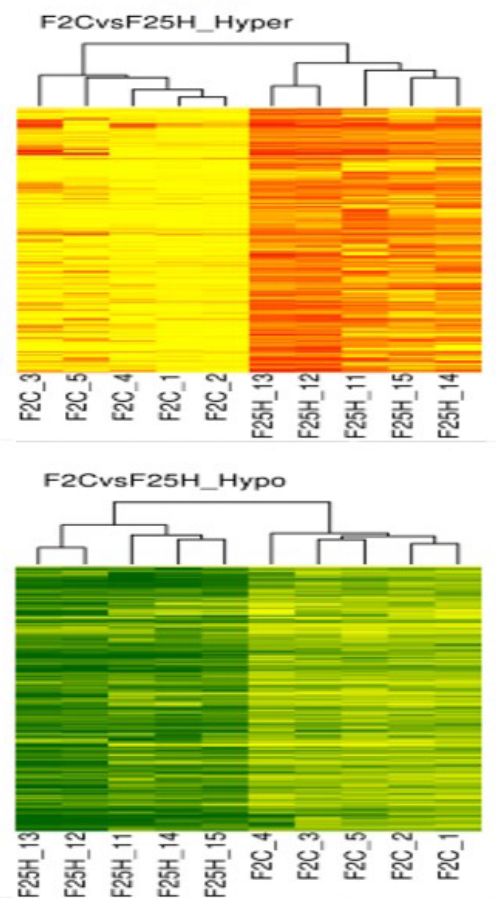

$\mathrm{CHH}$
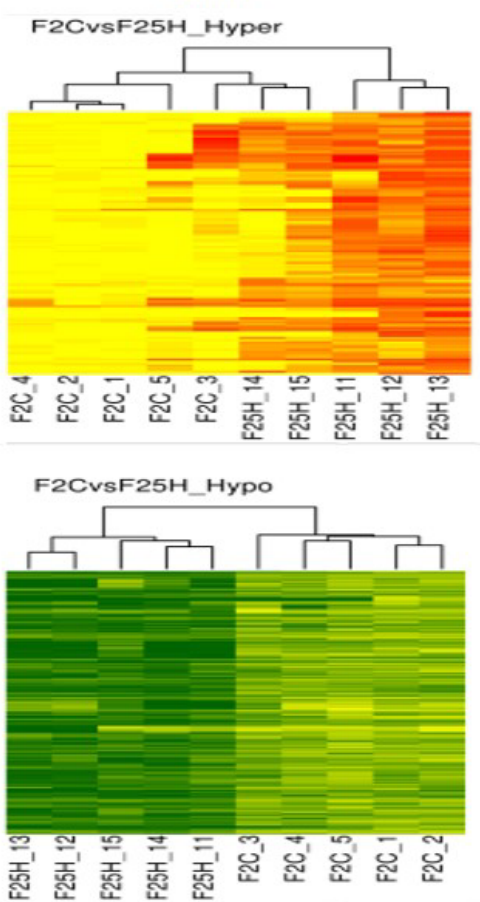

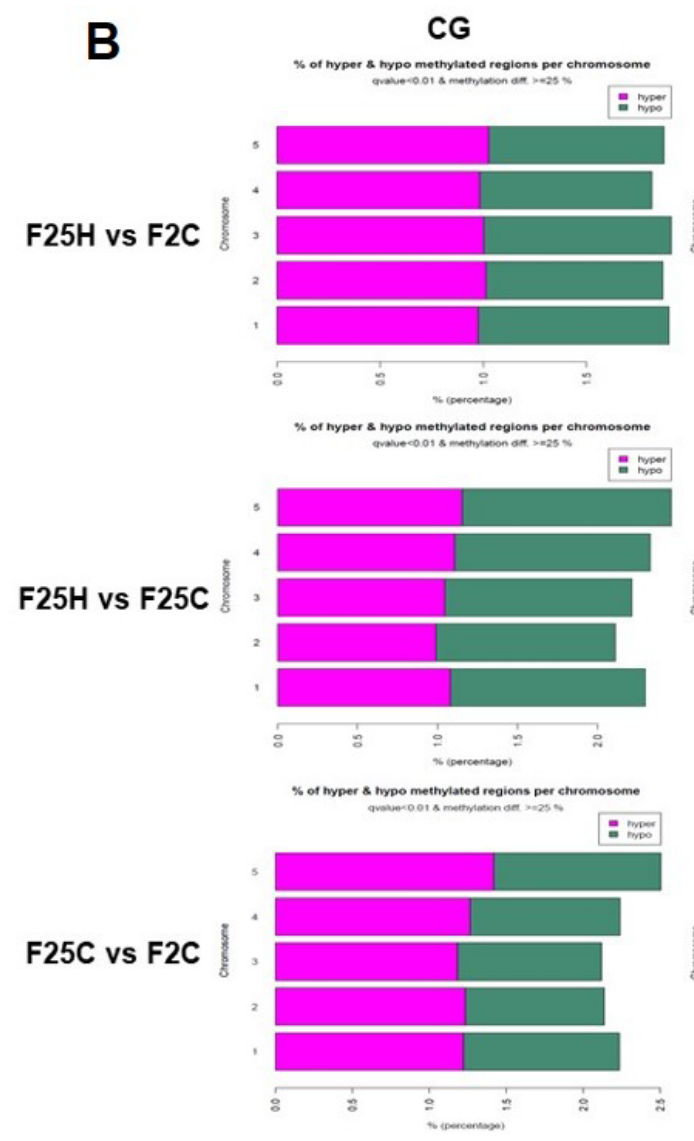

CHG

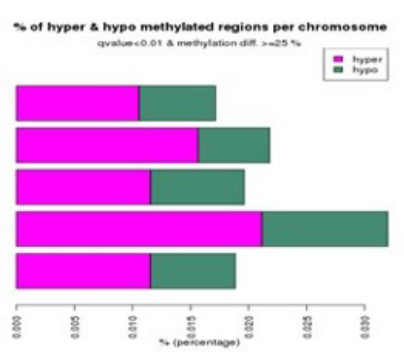

$\mathrm{CHH}$
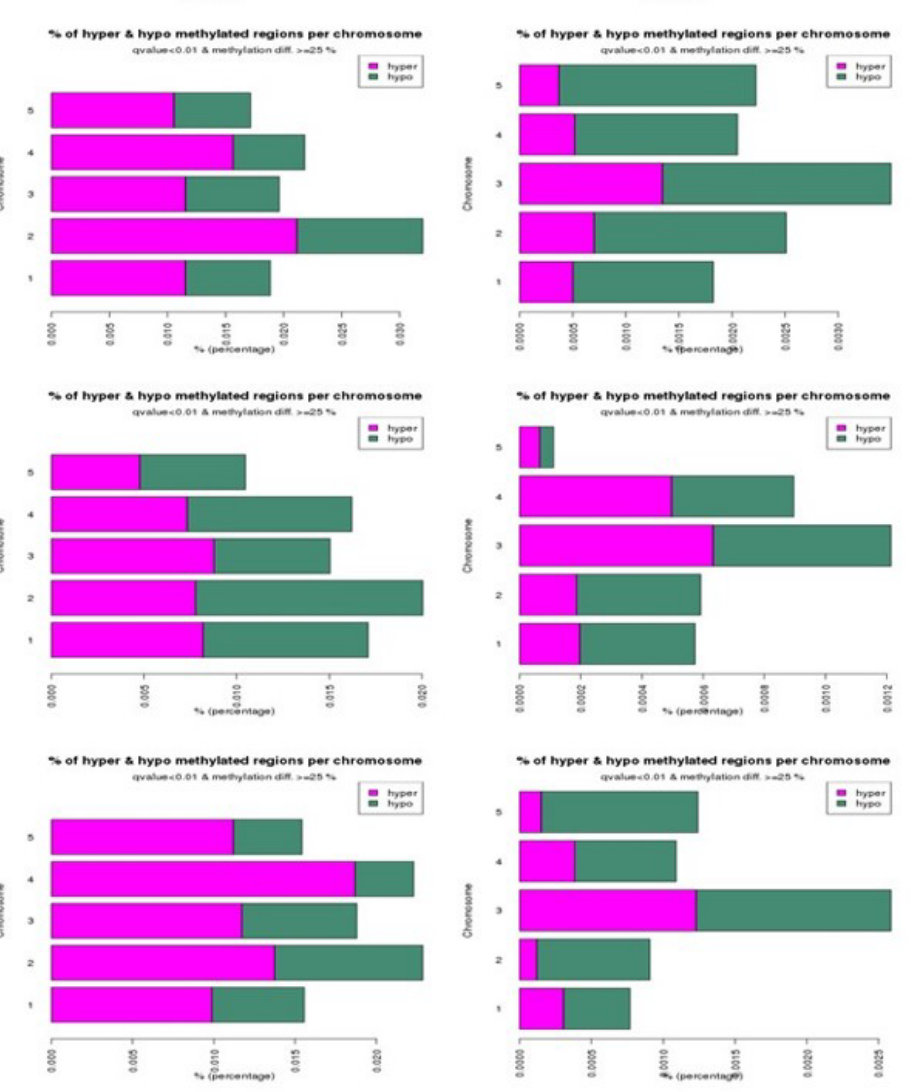
Figure 6. A hierarchical clustering heatmap analysis and the chromosomal distribution of DMPs (A) Heat maps of DMPs for hypermethylated cytosines (the upper panel) and hypomethylated cytosines (the lower panel) in $\mathrm{CG}, \mathrm{CHG}$ and $\mathrm{CHH}$ contexts in F25H vs. F2C. Differentially methylated cytosines in the genome with differences $>50 \%$ show the percentage of methylation in $\mathrm{F} 25 \mathrm{H}$ vs. F2C. In 'the upper panel', the red section indicates the larger percentage of methylation, and the yellow section indicates the lower percentage, and in 'the lower panel', the green section indicates the larger percentage of methylation and the yellow one indicates the lower percentage, q-value $<0.01$. (B) The percentage of distributions of hyper- and hypo- methylated DMPs through the chromosome level. DMPs in the CG context (the left panel), DMPs in the CHG context (the middle panel) and DMPs in the CHG context (the right panel) for each chromosome were identified in a 100-bp window. The pink section indicates the percentage of hypermethylation, and the green one indicates hypomethylation, q-value $<0.01$ and the methylation difference $>25 \%$.

\section{The distribution of DMPs and DMRs based on gene annotation}

DMPs and DMRs were then characterized to determine whether they were preferably located near genes. The location of hypo- or hyper- methylated DMRs was compared to the annotated Arabidopsis genes using genomation (Akalin et al., 2014).

In the CG context, the percentage of hypermethylated DMPs in all groups (F25H vs. $\mathrm{F} 2 \mathrm{C}, \mathrm{F} 25 \mathrm{H}$ vs. F25C and $\mathrm{F} 25 \mathrm{C}$ vs F2C) were the highest in the exon region (44\%, 51\% and $47 \%$, respectively), then in the promoter region $(33 \%, 27 \%$ and $30 \%$, respectively), followed by the intron region $(10 \%, 15 \%$ and $12 \%$, respectively) and the intergenic region $(13 \%, 7 \%$ and $11 \%$, respectively) (Figure 7A, C, E). Similarly, the percentage of hypomethylated DMPs sites were higher in gene bodies, especially in the exon regions followed by the promoter regions at the $\mathrm{CG}$ sites (Figure $7 \mathrm{~B}, \mathrm{D}, \mathrm{F})$. In the case of the $\mathrm{CHG}$ context, all groups $(\mathrm{F} 25 \mathrm{H}$ vs. F2C, F25H vs. F25C and F25C vs F2C) showed the highest percentage of hypermethylated DMPs in the promoter $(48 \%, 46 \%$ and $53 \%$, respectively), then in intergenic regions $(37 \%, 42 \%$ and $36 \%$, respectively), followed by the exon $(12 \%, 10 \%$ and $7 \%$, respectively) and the intron $(3 \%, 2 \%$ and 3\%, respectively) regions (Figure 7A, C, E). Interestingly, F25H vs. F2C groups showed the highest percentage of hypomethylated DMPs in the intergenic regions (48\%) followed by the promoter (44\%) compared with the F25C vs F2C groups where the highest percentage of DMPs was in the promoter region (52\%) followed by the intergenic regions (44\%) (Figure 7F). In the $\mathrm{CHH}$ context, in all groups (F25H vs. F2C, F25H vs. F25C and F25C vs F2C), the percentage of both hyper- and hypoDMPs was higher in the promoter (hyper: $88 \%, 76 \%$ and $77 \%$, respectively; hypo: $64 \%, 76 \%$ and $73 \%$, respectively), then in the intergenic regions (hyper: 10\%, 24\% and 22\%, 
respectively; hypo: $29 \%, 24 \%$ and $24 \%$, respectively) followed by the exon regions (hyper: $2 \%, 0 \%$ and $1 \%$, respectively; hypo: $6 \%, 0 \%$ and $3 \%$, respectively) and the intron regions (hyper: $0 \%, 0 \%$ and $0 \%$, respectively; hypo: 1\%,0\% and 0\%, respectively) (Figure 7).

Overall, in the case of the CG context, the highest percentage of DMPs was observed in the gene body, whereas in the case of the CHG and CHH contexts, it was highest in the promoter and intergenic regions. 

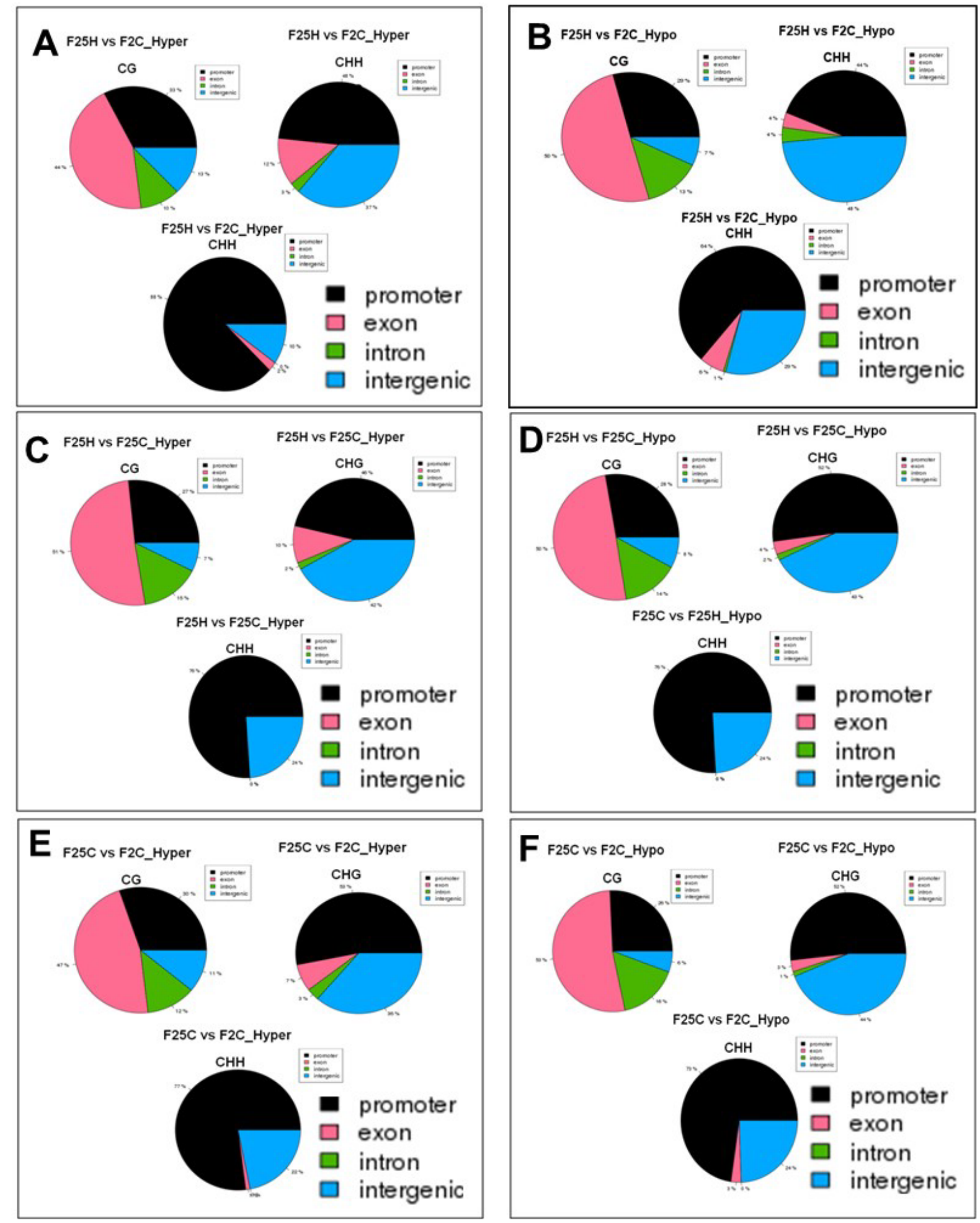

Figure 7. The distribution of hyper-and hypo-methylated DMPs in the genome in the comparison groups F25H vs. F2C (A - hyper, B - hypo), F25H vs F25C (C - hyper, D hypo) and F25C vs. F2C (E - hyper, F - hypo). The data are shown as an average percentage of differentially methylated cytosines at the promoter, exon, intron and intergenic regions. DMPs were considered as regions of methylation with $>25 \%$ differences. 
Comparative analysis revealed the heat-inducible nature of genetic variants and the spontaneous nature of epigenetic variants

The ratio between the number of epigenetic and genetic variations was $1.26 \mathrm{x}, 1.5 \mathrm{x}$ and $17 \mathrm{x}$ in the comparison groups of F25H vs. F2C, F25H vs. F25C and F25C vs F2C, respectively, indicating that epigenetic variations were more prevalent than genetic variations in the heatstressed vs control comparison groups. It was surprising that the largest difference was observed between F25C and F2C plants. It was initially expected that the difference between epigenetic and genetic variations would be the most drastic in the comparison groups involving $\mathrm{F} 25 \mathrm{H}$ plants. In contrast, the $\mathrm{F} 25 \mathrm{H}$ stressed progeny displayed 11 times more genetic variations than the $\mathrm{F} 25 \mathrm{C}$ parallel control progeny when both were compared with the parental control progeny F2C. Both these comparisons suggest that heat stress predominantly leads to the accumulation of genetic rather than epigenetic variations.

\begin{tabular}{|c|c|c|c|}
\hline \multirow{2}{*}{ Samples group } & $\begin{array}{c}\text { Epigenetic } \\
\text { variants }\end{array}$ & $\begin{array}{c}\text { Genetic } \\
\text { variants }\end{array}$ & $\begin{array}{c}\text { Epigenetic vs. } \\
\text { Genetic variants }\end{array}$ \\
\cline { 2 - 3 } & Total DMPs & $\begin{array}{c}\text { Total } \\
\text { (SNPs+INDELs) }\end{array}$ & \\
\hline F25H vs F2C & 66,491 & 52,753 & $1.26 \mathrm{x}$ \\
\hline F25H vs F25C & 78,412 & 52,896 & $1.5 \mathrm{x}$ \\
\hline F25C vs F2C & 80,464 & 4,672 & $17.0 \mathrm{x}$ \\
\hline
\end{tabular}

\section{Biological Enrichment Analysis}

The functional classification of variants (DMRs and DMPs) unique to each test group was interpreted using SuperViewer to identify regions with the statistically significant number of over-or under-represented genes and genomic features. Biological processes that might be enriched or under-represented within and between generations were assessed. All values were normalized by bootstrap x 100 , and p-values $<0.05$ were retrieved as significant. 
There was no difference in the enrichment of genetic variants for any biological process in F25H

The most enriched biological processes for SNPs was classed as 'unknown biological processes' regardless of the tested group (Figure S11). The only over-represented group was 'transport' found in F2C INDELs sample. All other biological processes were underrepresented for all samples, and no significant difference between the F25H group and any other group was observed (Supp. Figure S11, Table S1).

\section{Epimutation-associated genes participate in stress response pathways}

Gene Ontology (GO) analysis of epimutations (DMRs) in the hypermethylated CG context in F25H group showed a significant difference in the enrichment of biological processes in 'response to abiotic or biotic stimuli' and 'cell organization and biogenesis' compared to F2C and F25C groups (Supp. Figure S12A; Table S2). In the hypomethylated CG context, no significant difference was observed between the F25H vs F2C group and other groups (Supp. Figure S12B; Table S3).

In the hypomethylated $\mathrm{CHG}$ context, the $\mathrm{F} 25 \mathrm{H}$ vs $\mathrm{F} 2 \mathrm{C}$ group exhibited the enrichment in 'protein metabolism process' which was not observed in other groups (Supp. Figure S13A; Table S4). In the hypomethylated $\mathrm{CHH}$ context, the F25H vs F2C group exhibited the enrichment in 'transport', 'other metabolic processes', and 'developmental' processes (Supp. Figure S13B; Table S5).

\section{Discussion}

Being sessile, plants are more vulnerable to environmental changes. Plants face many environmental stresses such as abiotic stresses, including changes in light intensity, temperature fluctuations, nutrients and water availability, wind and other mechanical stimuli and biotic stresses, including various pathogens (Madlung and Comai 2004; Zhang et al. 2018). Environmental stresses have an impact on the directly exposed generation as well as on their progenies via parental effects and/ or transgenerational effects (Herman and Sultan 2011; Perez and Lehner 2019). Transgenerational effects refer exclusively to phenomena that could not be attributed to direct effects of a particular stress trigger on the affected organism. As such, only the altered phenotypes occurring in the second generation after a stress trigger can be truly described as transgenerational inheritance. Effects spanning shorter timescales are described as parental or intergenerational such as the altered phenotypes occurring in the immediate progeny generation after a trigger. However, many reports that described the 
intergenerational effects share mechanisms with transgenerational effects (Perez and Lehner 2019). The current study aimed to understand the effects of multigenerational exposure to heat stress on plant phenotypes, genotypes, and epigenotypes. Specifically, we compared the progeny of plants exposed to heat stress over 25 consecutive generations with their counterparts (the parental and parallel control progenies). We studied the genetic variations (SNPs and INDELs) by using the whole genome sequencing analysis as well as the epigenetic variations (DMPs and DMRs) by using the whole genome bisulfite sequencing analysis of the heat-stressed and control progenies. Overall, this study has deciphered the phenotypic, genomic and epigenomic resilience in the heat-stressed progeny in response to multigenerational exposure to heat stress.

\section{Multigenerational heat stress induced the phenotypic resilience in the stressed progeny}

The plant progeny phenotype can be affected by the parental environment (Groot et al. 2016). Although it has been reported that multigenerational exposure to stress changes the parental effects compared with single-generation exposures (otherwise known as transgenerational effects), multigenerational effects of stress exposure on the offspring phenotype elicit different outcomes that depend on dose effects and offspring environment effects (Groot et al. 2016). The current study explored parental effects on plant phenotypes and differences in response to multigenerational exposure to heat stress over twenty-five generations. Under heat stress, both the $\mathrm{F} 2 \mathrm{H}$ and $\mathrm{F} 25 \mathrm{H}$ stressed progenies survived better than their respective non-stressed parallel control progenies (Figure 1B). Our study showed that the progeny derived from 25 generations of heat stress displayed a notable stress tolerance at the mature stage which was slightly higher that the progeny exposed to heat stress for two generations (Figure 1B). Similar to our study, Wibowo et al. (2018) reported that the direct progeny of G2-G5 stressed plants exhibited the higher germination and survival rates under high-salinity stress, while the immediate G1 progeny did not, suggesting that the enhanced stress responses were only passed on after two consecutive generations of stress exposure but not when plants were exposed to hyperosmotic stress for only one episode. The authors concluded that stress priming requires repetitive exposure to stress. In some cases, it was evident that parental heat stress affected the response of offspring generation by providing evidence that heat stress accelerated leaf production in an attempt to speed up the development of the progeny of stressed plants (Liu et al. 2015; Porter 2005). Our previous studies showed that the heatinduced modifications of gene expression might have a role in phenotype changes such as leaf number and flowering time (Migicovsky et al. 2014). Zheng et al. (2017) also found that 
multigenerational exposure to drought improved the drought adaptability of rice offspring. The analysis of plant weight is useful for determining the active growth and development of a plant (Dornbos Jr and Mullen 1991; Yadav et al. 2012). F25H heat-stressed progeny plants displayed a higher fresh weight and dry weight when compared with parallel progeny plants.

The degree or severity of high temperature stress can impact cellular homeostasis (Kotak et al. 2007), and exposure to above the optimum growth temperature over time can result in the acquainted thermotolerance (Bray 2000; Sung et al. 2003). Moreover, the expression of transgenerational effects in the form of changes of phenotype responses strongly depends on the offspring environment (Boyko and Kovalchuk 2010; Groot et al. 2016) and varies between the genotype and trait (Suter and Widmer 2013a; Verhoeven and van Gurp 2012). Some reports have mentioned the effects of epigenetic inheritance or transference of transgenerational stress memory to be restricted to a single generation without the maintenance of stress (Pecinka et al. 2009; Suter and Widmer 2013b). Also, it was shown that multigenerational exposure often reduces the expression of parental effects compared to single-generation exposure (Groot et al. 2016).

Both intergenerational and transgenerational changes in response to stress include alterations in homologous recombination frequency (HRF). The progeny of plants that were often stressed show the elevated HRF (Migicovsky and Kovalchuk 2013). Under non-stress conditions, both the stressed progenies (F2H and F25H) exhibited the elevated HRF compared with their parallel control progenies (Figure 1C). It has been reported that plants exposed to salt and UV stresses show a much higher HRF than control plants (Boyko et al. 2010). The progeny of pathogen-infected plants have a higher level of somatic HRF, and the progeny also show the decreased methylation at loci for resistance to tobacco mosaic virus (TMV) and they have global hypermethylation (Boyko et al. 2007). Likewise, the progeny of plants exposed to heavy metal stress has high HRF (Rahavi et al. 2011). If stresses persist through multiple generations, it is possible that the epigenetic memory will be stronger and persist longer in the absence of stress, thus exhibiting changes in HRF for a longer period of time (Migicovsky and Kovalchuk 2013).

\section{The analysis of genomic variations induced by multi-generational exposure to heat stress}

In our study, we have shown that the progeny of heat-stressed plants had the higher frequency of genomic variations than the non-stressed control plants. It is well established that environmental stresses can be mutagenic and are capable of causing genome instability 
(Boyko et al. 2010; Boyko and Kovalchuk 2011; Gill et al. 2015). Environmental changes may also increase homologous recombination events or can facilitate the mobilization of transposable elements (Long et al. 2009; Boyko et al. 2010; Migicovsky and Kovalchuk 2013). Eventually, environmental conditions may induce mutations and increase the chances of genomic diversity leading to the adaptation to cope up with the ever-changing environmental conditions (Filichkin et al. 2010).

The position of SNPs may either influence the rate of transcription or alter the translation process depending on their corresponding genomic location such as exonic, intronic and untranslated regions; SNPs can also directly alter the coding sequence leading to a synonymous or non-synonymous amino acid replacement (Cingolani et al. 2012). SNPs and INDELs are vital genetic variations that can directly disrupt gene function and affect plant adaptability in the changing environment (Shastry 2009). For instance, SNPs can affect light response and flowering time by changing amino acids in phytochromes A and B (Filiault et al. 2008; Maloof et al. 2001). Jain et al. (2014) indicated that INDELs occur in different genomic regions, and they further suggested that positions of INDELs affect the function and expression of genes in a different manner. In the current study, genetic variants were mostly found in the upstream and downstream region of the gene and were significantly higher $(3 \mathrm{x})$ in the $\mathrm{F} 25 \mathrm{H}$ stressed progeny than in $\mathrm{F} 25 \mathrm{C}$ parallel and $\mathrm{F} 2 \mathrm{C}$ parental control progenies (Figure 2A, D). In contrast, the number of genetic variations was almost similar between the F25C parallel and F2C parental control progenies (Figure 2A). Positional profiles of these genetic variations (SNPs and INDELs) revealed the higher number of variant effects in the F25H heat-stressed progeny compared with the non-stressed parallel F25C and parental F2C progenies (Figure 3). The F25H heat-stressed progeny exhibited higher synonymous (12x) and nonsynonymous (6x) mutations than the parallel and parental control progenies (Figure 3). The non-synonymous mutations resulted in a biological variation in the living organism, consequently, they were subjected to a natural selection. Johannes et al. (2009) reported that flowering time and plant height in Arabidopsis was due to the stable inheritance of epialleles which included DNA insertion variants rather than accumulated a new epimutation linked to a phenotype trait. Although effects of variants were represented mostly as synonymous coding variants, a few ones were represented as non-synonymous variants (Cingolani et al. 2012). Zhang et al. (2013) found a frequent occurrence of SNPs in drought-resistance genes in common wheat. They also found that SNPs were associated with the genes responsible for the developmental processes and abiotic stress resistance in wheat (Zhang et al. 2013). SNPs can also create new splice sites and alter gene functions (Guyon-Debast et al. 2010). 
Therefore, the higher number of genetic variations in the heat-stressed progeny may be an indication of the adaptive processes occurring in the progeny of stressed plants.

Interestingly, the indels were of a similar size in $\mathrm{F} 25 \mathrm{C}$ and $\mathrm{F} 2 \mathrm{C}$, whereas they were significantly larger in F25H (Figure 2B), suggesting that multigenerational exposure to heat stress resulted in an increase in the size of INDELs in the heat-stressed progeny. Larger deletions may have a greater effect on gene expression and changes in the phenotype. It has been reported that small and large INDELs can cause pathogen sensitivity (Mindrinos et al. 1994; Kroymann et al. 2003).

The analysis of genes with SNPs identified in the progeny of stressed and nonstressed plants showed that the most enriched biological processes in SNPs was classed as "unknown biological processes". The biological processes such as "response to stress" and "response to abiotic or biotic stimulus" were commonly present in SNPs in F25H, F2C and F25C, with F25H having the higher enrichment in the biological processes such as "response to abiotic or biotic stimulus".

\section{The analysis of epigenetic variations induced by multi-generational exposure to heat stress}

The mechanism behind intergenerational and transgenerational stress memory in plants likely includes changes in the DNA sequence, DNA methylation and chromatin structure (Crisp et al. 2016; Zhang et al. 2018; Perez and Lehner 2019; Lind and Spagopoulou 2018; Miryeganeh and Saze 2020). DNA methylation is the most studied and best-narrated epigenetic modification involved in decoding the mechanisms of gene expression and the status of the chromatin structure (Pikaard and Mittelsten Scheid 2014; Miryeganeh and Saze 2020). Differential cytosine methylation, a form of heritable epigenetic polymorphism, underlies the phenotypic variation. The inheritance of changes in DNA methylation is one of the mechanisms of adaptation - epialleles often lead to the appearance of new alleles (Becker et al. 2011; Paszkowski and Grossniklaus 2011; Zhang et al. 2018 and reference therein). It has been reported that abiotic stresses can cause hyper- or hypomethylation in the genome after either short-term or long-term exposure (Uthup et al. 2011; Zhang et al. 2018 and reference therein). Several studies suggested that multigenerational stress exposure could cause a substantially higher heritable epigenetic variations compared with single-generation exposure (Remy 2010, Groot et al. 2016, Zheng et al. 2017). This phenomenon was explained by the gradual acclimatization of epigenetic effects (Groot et al. 2016). 
The genome-wide DNA methylome analysis revealed methylation levels of $24 \%$ in CG, $6.7 \%$ in $\mathrm{CHG}$ and $1.7 \%$ in $\mathrm{CHH}$ contexts in Arabidopsis thaliana which predominantly occur in transposons and other repetitive DNA elements (Cokus et al. 2008). In our study, the bisulfite sequencing data revealed that the average percentage of global genome methylation in the CG context was similar among all three groups (Figure 4A). However, in the CHG and $\mathrm{CHH}$ contexts, the $\mathrm{F} 25 \mathrm{H}$ stressed progeny showed a lower global methylation than the control progenies (Figure 4A). The data suggest that the reduction of methylation in the $\mathrm{CHG}$ $(p<0.1)$ and $\mathrm{CHH}(p<0.05)$ contexts might be a part of adaptation strategies to heat stress. It has been reported that abiotic stresses can cause hyper or hypomethylation in the genome after either short-term or long-term exposure (Thiebaut et al. 2019; Uthup et al. 2011). Hightemperature (HT) induced a global disruption of DNA methylation in cotton anther, mainly causing CHH hypomethylation in HT-sensitive cotton varieties (Ma et al. 2018). A decrease in global DNA methylation might result in the interruption of glucose- and reactive oxygen species (ROS)- producing metabolic pathways which may lead to microspore sterility (Ma et al. 2018). Min et al. (2014) also reported that in cotton anthers, high temperature decreased the expression of S-ADENOSYL-L-HOMOCYSTEINE HYDROLASE1 (SAHH1) and methyltransferases DRM1/DRM3, resulting in the genome-wide hypomethylation. Another report revealed that in soybean, heat stress induced the global DNA hypomethylation, especially in the $\mathrm{CHH}$ context, in both root hairs and stripped roots (Hossain et al. 2017). In cultured microspores of Brassica napus cv. Topas, heat-shock treatment $\left(32^{\circ} \mathrm{C}\right.$ for $\left.6 \mathrm{~h}\right)$ triggers DNA hypomethylation, particularly in the CG and CHG contexts (Li et al. 2016). However, Gao et al. (2014) reported that under heat stress $\left(37^{\circ} \mathrm{C}\right.$ for $2 \mathrm{~h}$, and then $45^{\circ} \mathrm{C}$ for 3h), global genome methylation levels were significantly higher in the heat-sensitive genotype of rapeseed than in the heat-tolerant genotype. Similarly, Arabidopsis and Cork oak plants also displayed an increased global DNA methylation levels under heat stress (Boyko et al. 2010; Correia et al. 2013). In Brassica rapa, the global DNA methylation levels are relatively stable under heat stress, while changes in $\mathrm{CHH}$ methylation at TE suggest that CHH methylation may be important for heat-stress response and tolerance (Liu et al., 2018). The single-base methylome analysis revealed that water deficit was associated with a decrease in $\mathrm{CHH}$ methylation in apple cultivars, which might result in the hypomethylated status of TEs (Xu et al., 2018). Wibowo et al. (2016) showed that methylation changes in $\mathrm{CHG}$ and $\mathrm{CHH}$ were well correlated with hyperosmotic stress treatment, whereas changes in CG methylation did not show any correlation, suggesting that CG methylation patterns occurred stochastically in the stressed and non-stressed samples. Further, they confirmed that 
hyperosmotic stress directed DNA methylation changes primarily at non-CG sites, and these epigenetic modifications were associated with an acquired transient adaptation to stress. Exposure to abiotic stressors such as salt also causes DNA hypomethylation in the progeny; Jeremias et al. (2018) reported the inheritance of DNA hypomethylation in response to salinity stress in Daphnia magna. Also, Jiang et al. (2014) showed that lineages exposed to soil salinity stress accumulated more methylation at the CG sites than the control progenies. Epigenomic changes have been observed in response to salt treatment in the offspring of the exposed plants in rice and Arabidopsis thaliana (Bilichak et al. 2012; Karan et al. 2012). Likewise, distinct DNA methylation patterns have been observed in response to environmental signals such as heat and salt (Yao et al. 2012; Popova et al. 2013). Recently, Atighi et al. (2020) showed a massive and genome-wide hypomethylation as a crucial plant defence mechanism in response to nematode or bacterial pathogen infection in rice and tomato. Further, they demonstrated that DNA hypomethylation in the $\mathrm{CHH}$ context was associated with a reduced susceptibility to root-parasitic nematodes in rice. Although, exposure to bacterial pathogen Pseudomonas syringae led to hypomethylation in the genomic regions associated with plant defence genes and in the pericentromeric regions in Arabidopsis; accordingly, methylation-deficient mutants were less susceptible to this pathogen (Pavet et al. 2006). Similarly, the hypomethylated Arabidopsis mutants showed a higher resistance to pathogen Hyaloperonospora arabidopsidis (Lopez Sanchez et al. 2016). Furthermore, the Arabidopsis demethylase triple mutant (ros $1 / d m l 2 / d m l 3$ ) exhibited an increased susceptibility to infection by Fusarium oxysporum (Le et al. 2014).

Hierarchical clustering of epimutations and the heat map analysis of changes in both hypo- and hypermethylated DMPs revealed that the F25H progeny of stressed plants showed a clear separation from plants of the parental and parallel control progenies (Figure 5D, 6A), indicating directional epimutations in the $\mathrm{F} 25 \mathrm{H}$ stressed progeny due to multigenerational exposure to heat stress. Ganguly et al. (2017) also found similar clustering patterns in response to heat. They showed that in Arabidopsis, the progeny of stressed plants clustered separately from the progeny of non-stressed plants in response to the treatment with heat stress (Ganguly et al. 2017). Becker et al. (2011) compared global DNA methylation patterns among 10 Arabidopsis thaliana lines propagated for 30 generations from a common ancestor. Hierarchical clustering based on DMPs grouped siblings of the 3rd and 31st generation lines together in separate groups and suggested that epimutations did not distribute randomly throughout the genome (Becker et al. 2011). Similar to our study, Zheng et al. (2017) showed that the multi-generational drought stress induced directional epimutations in the methylome 
of rice offspring. Eichten and Springer (2015) reported that the separate hierarchical clustering of epimutations was associated with cold stress treatment.

We observed that the total number of differentially methylated positions (DMPs) was higher between the control groups F25C vs. F2C $(80,464)$ compared with the stressed progeny groups F25H vs. F2C $(66,491)$ and F25H vs. F25C $(78,412)$ (Figure 4B, C). It was somewhat surprising because we expected an opposite result; we hypothesized that heat stress would induce epimutations and expected to see a higher number of epigenomic variations in the progeny of heat stressed plants compared with the controls. It is possible that heat stress does not result in an increase in epimutations, but it rather causes epimutations to occur at the specific, heat stress-related sites in the genome. The fact that the observed progenies of heatstressed plants cluster together confirms this hypothesis. Becker et al. (2011) have compared the genome wide DNA methylation patterns between the $31^{\text {st }}$ generation (that grew under control conditions) and the ancestor generation (the3rd) and deciphered 30,000 differentially methylated cytosines (DMPs). More than one third of recurrent events were observed in the whole DMPs and DMRs, indicating the non-random nature of these epimutations. Further, they reported an average of 3,300 DMPs between immediate generations; this was around three times more than what would have been expected from 30,000 DMPs accumulated between the 3rd and the 31st generation lines (Becker et al. 2011). This data suggest that the number of epimutations does not increase linearly with time, indicating that only a fraction of new DMPs is maintained by the transgenerational inheritance over the longer term (Becker et al. 2011).

The analysis of DMPs in a specific sequence context revealed a different pattern. While DMPs in the CG context were higher in the F25C vs. F2C control group, in the CHG and $\mathrm{CHH}$ context, DMPs were higher in comparison groups involving the $\mathrm{F} 25 \mathrm{H}$ stressed progeny (Figure 4C). The F25H vs. F2C comparison group showed the higher number of hypomethylated and hypermethylated DMPs in the $\mathrm{CHG}$ and $\mathrm{CHH}$ contexts compared with the control comparison groups (Figure 4C). These data suggest that the epimutations induced by multigenerational exposure to heat stress are primarily associated with changes in the CHG and $\mathrm{CHH}$ contexts, and that hypomethylation is a prevalent mechanism. A similar phenomenon has been narrated in response to heavy metal stresses where it has been reported that hypomethylation prevails at several loci in hemp and clover (Aina et al. 2004). In epigenetic mutants, methylation patterns of DMPs link DNA methylation to the response to the environmental signals like heat stress (Popova et al. 2013) and salt stress (Yao et al. 2012). Several reports also suggest that it is common for plants to methylate cytosines in the 
sequence contexts of $\mathrm{CHG}$ and $\mathrm{CHH}$, but the reestablishment of epigenetic modifications is mostly guided by small RNAs or the heterochromatin-directed methylation pathways (Feng, Jacobsen et al. 2010, Stroud et al. 2013). Further, we observed that in the case of the CG context, the highest percentage of DMPs was observed in gene body, whereas in the case of the $\mathrm{CHG}$ and $\mathrm{CHH}$ contexts, it was the highest in the promoter and intergenic regions. Gene body methylation was found within coding sequences with a potential to contribute to phenotypes (Bewick and Schmitz 2017), and in promoters and first exons, the CG sequences were usually found to cluster around the regulatory region of genes (Zheng et al. 2013). It was reported before that the promoters of stress-responsive genes may be hypomethylated under stress conditions (Yao and Kovalchuk 2011, Bilichak et al. 2012).

The Gene Ontology analysis identified that the F25H stressed progeny epimutations showed enrichment in the stress-related pathways such as 'response to stress' and 'response to abiotic or biotic stimuli' (Figure S12A; Table S2). Liu et al. (2018) found the enrichment of heat-induced DMRs in the RNA metabolism and modification process, which may suggest that the fine-tuning mechanism of DNA methylation in regulating the corresponding functional genes handles the influence of heat stress over time. Zheng et al. (2017) reported that in rice, the long-term drought stress-induced epimutations were directly involved in the stress-responsive pathways. Wibowo et al. (2016) highlighted that hyperosmotic-stress induced DMRs were enriched with functions related to metabolic responses and ion transport, and he also suggested that exposure to hyperosmotic-stress targeted the discrete and epigenetically labile regions of the genome. The DMR-associated genes in the droughttolerant introgression line DK151 are mainly involved in stress response, programmed cell death, and the nutrient reservoir activity, which may contribute to the constitutive drought tolerance (Wang et al., 2016).

In conclusion, the current study explored the effects of exposure to multigenerational heat stress in A. thaliana. The stressed progeny derived from 25 generations of heat stress displayed a notable stress tolerance at the mature stage. The comparative genomic and epigenomic analysis revealed that the $\mathrm{F} 25 \mathrm{H}$ heat-stressed lineage showed the higher number of genetic mutations (SNPs and INDELs) and epimutations (only in the $\mathrm{CHG}$ and $\mathrm{CHH}$ context) compared with the parallel (F25C) and parental (F2C) lineages, suggesting that heat stress induced the genetic and epigenetic variations. The F25H heat-stressed progeny showed a 7-fold higher number of non-synonymous mutations than the non-stressed parental line, which might result in changes in phenotype. Hierarchical clustering of epimutations separated the heat-stressed and control parental progenies into distinct groups which revealed the non- 
random nature of heat-induced epimutations. The Gene Ontology analysis revealed that mutations and epimutations were enriched in the stress-related pathways such as 'response to stress' and 'response to abiotic or biotic stimuli'. Overall, our study suggests that the genetic and epigenetic mutations induced by heat stress in the progeny might result in a phenotypic resilience to adverse environments and potentially trigger to the process of microevolution under long-term stress conditions.

\section{Acknowledgements}

We thank Natural Sciences and Engineering Research Council of Canada (NSERC) for funding our work. Prof. Gideon Grafi from Ben-Gurion University of the Negev, Israel, has been acknowledged for scientific input on the manuscript first draft.

\section{Author contributions}

Narendra Singh Yadav- designed and performed experiments, analyzed data, wrote the manuscript

Viktor Titov- performed experiments and analyzed the data

Ivie Ayemere- performed experiments and analyzed the data

Boseon Byeon- conducted bioinformatics analysis

Yaroslav Ilnytskyy- conducted bioinformatics analysis

Igor Kovalchuk- came up with the concept of the study, analyzed data and contributed to writing.

\section{Competing interests}

The authors declare that they have no competing interests.

\section{Materials \& Correspondence.}

Indicate the author(s) to whom correspondence and material requests should be addressed.

\section{References}

Aina, R., Sgorbati, S., Santagostino, A., Labra, M., Ghiani, A., \& Citterio, S. (2004). Specific hypomethylation of DNA is induced by heavy metals in white clover and industrial hemp. Physiologia Plantarum, 121(3), 472-480. 
Akalin, A., Franke, V., Vlahoviček, K., Mason, C. E., \& Schübeler, D. (2014). Genomation: a toolkit to summarize, annotate and visualize genomic intervals. Bioinformatics, 31(7), 11271129.

Akalin, A., Kormaksson, M., Li, S., Garrett-Bakelman, F. E., Figueroa, M. E., Melnick, A., \& Mason, C. E. (2012). methylKit: a comprehensive R package for the analysis of genome-wide DNA methylation profiles. Genome Biology, 13(10), R87.

Atighi, M. R., Verstraeten, B., De Meyer, T., \& Kyndt, T. (2020). Genome $\square$ wide DNA hypomethylation shapes nematode pattern $\square$ triggered immunity in plants. New Phytologist, 227(2), 545-558.

Banerjee, A., Wani, S. H., \& Roychoudhury, A. (2017). Epigenetic control of plant cold responses. Frontiers in Plant Science, 8, 1643.

Becker, C., Hagmann, J., Müller, J., Koenig, D., Stegle, O., Borgwardt, K., \& Weigel, D. (2011). Spontaneous epigenetic variation in the Arabidopsis thaliana methylome. Nature, 480(7376), 245-249.

Bewick, A. J., \& Schmitz, R. J. (2017). Gene body DNA methylation in plants. Current opinion in plant biology, 36, 103-110.

Bilichak, A., \& Kovalchuk, I. (2016). Transgenerational response to stress in plants and its application for breeding. Journal of Experimental Botany, 67(7), 2081-2092.

Bilichak, A., Ilnystkyy, Y., Hollunder, J., \& Kovalchuk, I. (2012). The progeny of Arabidopsis thaliana plants exposed to salt exhibit changes in DNA methylation, histone modifications and gene expression. PloS one, 7(1), e30515.

Bilichak, A., Ilnytskyy, Y., Wóycicki, R., Kepeshchuk, N., Fogen, D., \& Kovalchuk, I. (2015). The elucidation of stress memory inheritance in Brassica rapa plants. Frontiers in plant science, $6,5$.

Boyko, A., \& Kovalchuk, I. (2010). Transgenerational Response to stress in Arabidopsis thaliana. Plant signaling \& behavior, 5(8), 995-998.

Boyko, A., \& Kovalchuk, I. (2011). Genome instability and epigenetic modificationheritable responses to environmental stress?. Current opinion in plant biology, 14(3), 260266.

Boyko, A., Blevins, T., Yao, Y., Golubov, A., Bilichak, A., Ilnytskyy, Y., ... \& Kovalchuk, I. (2010). Transgenerational adaptation of Arabidopsis to stress requires DNA methylation and the function of Dicer-like proteins. PloS one, 5(3), e9514.

Boyko, A., Kathiria, P., Zemp, F. J., Yao, Y., Pogribny, I., \& Kovalchuk, I. (2007). Transgenerational changes in the genome stability and methylation in pathogen-infected plants: (Virus-induced plant genome instability). Nucleic acids research, 35(5), 1714-1725.

Bray, E. A. (2000). Response to abiotic stress. Biochemistry and molecular biology of plants, 1158-1203.

Chinnusamy, V., Schumaker, K., \& Zhu, J. K. (2004). Molecular genetic perspectives on cross $\square$ talk and specificity in abiotic stress signalling in plants. Journal of Experimental Botany, 55(395), 225-236. 
Cingolani, P., Platts, A., Wang, L. L., Coon, M., Nguyen, T., Wang, L., ... \& Ruden, D. M. (2012). A program for annotating and predicting the effects of single nucleotide polymorphisms, SnpEff: SNPs in the genome of Drosophila melanogaster strain w1118; iso2; iso-3. Fly, 6(2), 80-92.

Cokus, S. J., Feng, S., Zhang, X., Chen, Z., Merriman, B., Haudenschild, C. D., ... \& Jacobsen, S. E. (2008). Shotgun bisulphite sequencing of the Arabidopsis genome reveals DNA methylation patterning. Nature, 452(7184), 215-219.

Correia, B., Valledor, L., Meijon, M., Rodriguez, J. L., Dias, M. C., Santos, C., ... \& Pinto, G. (2013). Is the interplay between epigenetic markers related to the acclimation of cork oak plants to high temperatures?. PLoS One, 8(1), e53543.

Crisp, P. A., Ganguly, D., Eichten, S. R., Borevitz, J. O., \& Pogson, B. J. (2016). Reconsidering plant memory: Intersections between stress recovery, rna turnover, and epigenetics. Science advances, 2(2), e1501340.

Dong, B. C., Yu, F. H., \& Roiloa, S. R. (2019). Ecoepigenetics in Clonal and Inbreeding Plants: Transgenerational Adaptation and Environmental Variation. Frontiers in plant science, 10,622 .

Dornbos Jr, D., \& Mullen, R. (1991). Influence of stress during soybean seed fill on seed weight, germination, and seedling growth rate. Canadian Journal of Plant Science, 71(2), 373-383.

Dowen, R. H., Pelizzola, M., Schmitz, R. J., Lister, R., Dowen, J. M., Nery, J. R., ... \& Ecker, J. R. (2012). Widespread dynamic DNA methylation in response to biotic stress. Proceedings of the National Academy of Sciences, 109(32), E2183-E2191.

Duempelmann, L., Skribbe, M., \& Bühler, M. (2020). Small RNAs in the Transgenerational Inheritance of Epigenetic Information. Trends in Genetics, 36(3), 203-214.

Eichten, S. R., \& Springer, N. M. (2015). Minimal evidence for consistent changes in maize DNA methylation patterns following environmental stress. Frontiers in plant science, 6, 308.

Eichten, S. R., Schmitz, R. J., \& Springer, N. M. (2014). Epigenetics: beyond chromatin modifications and complex genetic regulation. Plant Physiology, 165(3), 933-947.

Feng, S., Jacobsen, S. E., \& Reik, W. (2010). Epigenetic reprogramming in plant and animal development. Science, 330(6004), 622-627.

Filiault, D. L., Wessinger, C. A., Dinneny, J. R., Lutes, J., Borevitz, J. O., Weigel, D., ... \& Maloof, J. N. (2008). Amino acid polymorphisms in Arabidopsis phytochrome B cause differential responses to light. Proceedings of the National Academy of Sciences, 105(8), 3157-3162.

Filichkin, S. A., Priest, H. D., Givan, S. A., Shen, R., Bryant, D. W., Fox, S. E., ... \& Mockler, T. C. (2010). Genome-wide mapping of alternative splicing in Arabidopsis thaliana. Genome research, 20(1), 45-58.

Ganguly, D., Crisp, P. A., Eichten, S. R., \& Pogson, B. J. (2017). The Arabidopsis DNA methylome is stable under transgenerational drought stress. Plant Physiology, 175(4), 18931912. 
Gao, G., Li, J., Li, H., Li, F., Xu, K., Yan, G., ... \& Wu, X. (2014). Comparison of the heat stress induced variations in DNA methylation between heat-tolerant and heat-sensitive rapeseed seedlings. Breeding science, 64(2), 125-133.

Gill, S. S., Anjum, N. A., Gill, R., Jha, M., \& Tuteja, N. (2015). DNA damage and repair in plants under ultraviolet and ionizing radiations. The Scientific World Journal, 2015.

Gouvêa, D. Y., Aprison, E. Z., \& Ruvinsky, I. (2015). Experience Modulates the Reproductive Response to Heat Stress in C. elegans via Multiple Physiological Processes. PloS one, 10(12), e0145925.

Groot, M. P., Kooke, R., Knoben, N., Vergeer, P., Keurentjes, J. J., Ouborg, N. J., \& Verhoeven, K. J. (2016). Effects of multi-generational stress exposure and offspring environment on the expression and persistence of transgenerational effects in Arabidopsis thaliana. PloS one, 11(3), e0151566.

Gutzat, R., \& Scheid, O. M. (2012). Epigenetic responses to stress: triple defense?. Current Opinion in Plant Biology, 15(5), 568-573.

Guyon-Debast, A., Lécureuil, A., Bonhomme, S., Guerche, P., \& Gallois, J. L. (2010). A SNP associated with alternative splicing of RPT5b causes unequal redundancy between RPT5a and RPT5b among Arabidopsis thaliananatural variation. BMC plant biology, 10(1), 158.

Haque, M. M., Nilsson, E. E., Holder, L. B., \& Skinner, M. K. (2016). Genomic Clustering of differential DNA methylated regions (epimutations) associated with the epigenetic transgenerational inheritance of disease and phenotypic variation. BMC Genomics, 17(1), 418.

Herman, J. J., \& Sultan, S. E. (2011). Adaptive transgenerational plasticity in plants: case studies, mechanisms, and implications for natural populations. Frontiers in plant science, 2, 102.

Hossain, M. S., Kawakatsu, T., Kim, K. D., Zhang, N., Nguyen, C. T., Khan, S. M., ... \& Schmitz, R. J. (2017). Divergent cytosine DNA methylation patterns in single $\square$ cell, soybean root hairs. New Phytologist, 214(2), 808-819.

Huang, G. T., Ma, S. L., Bai, L. P., Zhang, L., Ma, H., Jia, P., ... \& Guo, Z. F. (2012). Signal transduction during cold, salt, and drought stresses in plants. Molecular biology reports, 39(2), 969-987.

Ilnytskyy, Y., Boyko, A., \& Kovalchuk, I. (2004). Luciferase-based transgenic recombination assay is more sensitive than $\beta$-glucoronidase-based. Mutation Research/Genetic Toxicology and Environmental Mutagenesis, 559(1-2), 189-197.

Jain, M., Moharana, K. C., Shankar, R., Kumari, R., \& Garg, R. (2014). Genomewide discovery of DNA polymorphisms in rice cultivars with contrasting drought and salinity stress response and their functional relevance. Plant Biotechnology Journal, 12(2), 253-264.

Jang, S. J., Sato, M., Sato, K., Jitsuyama, Y., Fujino, K., Mori, H., ... \& Abe, J. (2015). A single-nucleotide polymorphism in an endo-1, 4- $\beta$-glucanase gene controls seed coat permeability in soybean. PLoS One, 10(6), e0128527. 
Jeremias, G., Barbosa, J., Marques, S. M., De Schamphelaere, K. A., Van Nieuwerburgh, F., Deforce, D., ... \& Asselman, J. (2018). Transgenerational inheritance of DNA hypomethylation in Daphnia magna in response to salinity stress. Environmental science \& technology, 52(17), 10114-10123.

Jiang, C., Mithani, A., Belfield, E. J., Mott, R., Hurst, L. D., \& Harberd, N. P. (2014). Environmentally responsive genome-wide accumulation of de novo Arabidopsis thaliana mutations and epimutations. Genome research, 24(11), 1821-1829.

Johannes, F., \& Schmitz, R. J. (2019). Spontaneous epimutations in plants. New Phytologist, 221(3), 1253-1259.

Johannes, F., Porcher, E., Teixeira, F. K., Saliba-Colombani, V., Simon, M., Agier, N., ... \& Bouchez, D. (2009). Assessing the impact of transgenerational epigenetic variation on complex traits. PLoS Genet, 5(6), e1000530.

Karan, R., DeLeon, T., Biradar, H., \& Subudhi, P. K. (2012). Salt stress induced variation in DNA methylation pattern and its influence on gene expression in contrasting rice genotypes. PloS one, 7(6), e40203.

Kotak, S., Larkindale, J., Lee, U., von Koskull-Döring, P., Vierling, E., \& Scharf, K.-D. (2007). Complexity of the heat stress response in plants. Current opinion in plant biology, $10(3), 310-316$.

Kovalchuk, I., \& Kovalchuk, O. (2012). Epigenetics in Health and Disease (Paperback). FT Press.

Kroymann, J., Donnerhacke, S., Schnabelrauch, D., \& Mitchell-Olds, T. (2003). Evolutionary dynamics of an Arabidopsis insect resistance quantitative trait locus. Proceedings of the National Academy of Sciences, 100 (suppl 2), 14587-14592.

Krueger, F., \& Andrews, S. R. (2011). Bismark: a flexible aligner and methylation caller for Bisulfite-Seq applications. Bioinformatics, 27(11), 1571-1572.

Lane, A. K., Niederhuth, C. E., Ji, L., \& Schmitz, R. J. (2014). pENCODE: a plant encyclopedia of DNA elements. Annual Review of Genetics, 48, 49-70.

Le, T. N., Schumann, U., Smith, N. A., Tiwari, S., Au, P. C. K., Zhu, Q. H., ... \& Dennis, E. S. (2014). DNA demethylases target promoter transposable elements to positively regulate stress responsive genes in Arabidopsis. Genome biology, 15(9), 458.

Li, J., Huang, Q., Sun, M., Zhang, T., Li, H., Chen, B., ... \& Qiao, J. (2016). Global DNA methylation variations after short-term heat shock treatment in cultured microspores of Brassica napus cv. Topas. Scientific reports, 6, 38401.

Liégard, B., Baillet, V., Etcheverry, M., Joseph, E., Lariagon, C., Lemoine, J., ... \& Jubault, M. (2019). Quantitative resistance to clubroot infection mediated by transgenerational epigenetic variation in Arabidopsis. New Phytologist, 222(1), 468-479.

Lind, M. I., \& Spagopoulou, F. (2018). Evolutionary consequences ofepigenetic inheritance. Heredity 121(3), 205-209. 
Liu, G., Xia, Y., Liu, T., Dai, S., \& Hou, X. (2018). The DNA methylome and association of differentially methylated regions with differential gene expression during heat stress in Brassica rapa. International journal of molecular sciences, 19(5), 1414.

Liu, J., Feng, L., Li, J., \& He, Z. (2015). Genetic and epigenetic control of plant heat responses. Frontiers in Plant Science, 6, 267-267.

Long, L., Ou, X., Liu, J., Lin, X., Sheng, L., \& Liu, B. (2009). The spaceflight environment can induce transpositional activation of multiple endogenous transposable elements in a genotype-dependent manner in rice. Journal of plant physiology, 166(18), 2035-2045.

López Sánchez, A., Stassen, J. H., Furci, L., Smith, L. M., \& Ton, J. (2016). The role of DNA (de) methylation in immune responsiveness of Arabidopsis. The Plant Journal, 88(3), 361374.

Ma, Y., Min, L., Wang, M., Wang, C., Zhao, Y., Li, Y., ... \& Su, X. (2018). Disrupted genome methylation in response to high temperature has distinct affects on microspore abortion and anther indehiscence. The Plant Cell, 30(7), 1387-1403.

Madlung, A., \& Comai, L. (2004). The effect of stress on genome regulation and structure. Annals of Botany, 94(4), 481-495.

Maloof, J. N., Borevitz, J. O., Dabi, T., Lutes, J., Nehring, R. B., Redfern, J. L., ... \& Weigel, D. (2001). Natural variation in light sensitivity of Arabidopsis. Nature genetics, 29(4), 441446.

Manikkam, M., Guerrero-Bosagna, C., Tracey, R., Haque, M. M., \& Skinner, M. K. (2012). Transgenerational actions of environmental compounds on reproductive disease and identification of epigenetic biomarkers of ancestral exposures. PloS one, 7(2), e31901.

Migicovsky, Z., \& Kovalchuk, I. (2013). Changes to DNA methylation and homologous recombination frequency in the progeny of stressed plants. Biochemistry and Cell Biology, 91(1), 1-5.

Migicovsky, Z., \& Kovalchuk, I. (2015). Transgenerational inheritance of epigenetic response to cold in Arabidopsis thaliana. Biocatalysis and Agricultural Biotechnology, 4(1), $1-10$.

Migicovsky, Z., Yao, Y., \& Kovalchuk, I. (2014). Transgenerational phenotypic and epigenetic changes in response to heat stress in Arabidopsis thaliana. Plant signaling \& behavior, 9(2), e27971.

Min, L., Li, Y., Hu, Q., Zhu, L., Gao, W., Wu, Y., ... \& Zhang, X. (2014). Sugar and auxin signaling pathways respond to high-temperature stress during anther development as revealed by transcript profiling analysis in cotton. Plant Physiology, 164(3), 1293-1308.

Mindrinos, M., Katagiri, F., Yu, G. L., \& Ausubel, F. M. (1994). The Arabidopsis thaliana disease resistance gene RPS2 encodes a protein containing a nucleotide-binding site and leucine-rich repeats. Cell, 78(6), 1089-1099.

Mirouze, M., \& Paszkowski, J. (2011). Epigenetic contribution to stress adaptation in plants. Current Opinion in Plant Biology, 14(3), 267-274. 
Miryeganeh, M., \& Saze, H. (2020). Epigenetic inheritance and plant evolution. Population Ecology, 62(1), 17-27.

Miura, K., Agetsuma, M., Kitano, H., Yoshimura, A., Matsuoka, M., Jacobsen, S. E., \& Ashikari, M. (2009). A metastable DWARF1 epigenetic mutant affecting plant stature in rice. Proceedings of the National Academy of Sciences, 106(27), 11218-11223.

Murgia, I., Giacometti, S., Balestrazzi, A., Paparella, S., Pagliano, C., \& Morandini, P. (2015). Analysis of the transgenerational iron deficiency stress memory in Arabidopsis thaliana plants. Frontiers in plant science, 6, 745.

Nicotra, A. B., Atkin, O. K., Bonser, S. P., Davidson, A. M., Finnegan, E., Mathesius, U., . . . \& Valladares, F. (2010). Plant phenotypic plasticity in a changing climate. Trends in Plant Science, 15(12), 684-692.

Norouzitallab, P., Baruah, K., Vandegehuchte, M., Van Stappen, G., Catania, F., Bussche, J. V., . . \& \& Bossier, P. (2014). Environmental heat stress induces epigenetic transgenerational inheritance of robustness in parthenogenetic Artemia model. The FASEB Journal, 28(8), 3552-3563.

Ou, X., Zhang, Y., Xu, C., Lin, X., Zang, Q., Zhuang, T., . . . \& Liu, B. (2012). Transgenerational inheritance of modified DNA methylation patterns and enhanced tolerance induced by heavy metal stress in rice (Oryza sativa L.). PloS one, 7(9), e41143.

Paszkowski, J., \& Grossniklaus, U. (2011). Selected aspects of transgenerational epigenetic inheritance and resetting in plants. Current opinion in plant biology, 14(2), 195-203.

Pavet, V., Quintero, C., Cecchini, N. M., Rosa, A. L., \& Alvarez, M. E. (2006). Arabidopsis displays centromeric DNA hypomethylation and cytological alterations of heterochromatin upon attack by Pseudomonas syringae. Molecular Plant-Microbe Interactions, 19(6), 577 587.

Pecinka, A., Rosa, M., Schikora, A., Berlinger, M., Hirt, H., Luschnig, C., \& Mittelsten Scheid, O. (2009). Transgenerational stress memory is not a general response in arabidopsis. PloS one, 4(4), e5202.

Perez, M. F., \& Lehner, B. (2019). Intergenerational and transgenerational epigenetic inheritance in animals. Nature cell biology, 21(2), 143-151.

Pikaard, C. S., \& Scheid, O. M. (2014). Epigenetic regulation in plants. Cold Spring Harbor perspectives in biology, 6(12), a019315.

Popova, O. V., Dinh, H. Q., Aufsatz, W., \& Jonak, C. (2013). The rddm pathway is required for basal heat tolerance in arabidopsis. Molecular Plant, 6(2), 396-410.

Porter, J. R. (2005). Rising temperatures are likely to reduce crop yields. Nature, 436(7048), 174.

Provart, N., \& Zhu, T. (2003). A browser-based functional classification SuperViewer for Arabidopsis genomics. Currents in Computational Molecular Biology, 2003, 271-272.

Rahavi, S. M., \& Kovalchuk, I. (2013). Changes in homologous recombination frequency in Arabidopsis thaliana plants exposed to stress depend on time of exposure during development 
and on duration of stress exposure. Physiology and Molecular Biology of Plants, 19(4), 479488. doi:10.1007/s12298-013-0197-z

Ramírez-Carrasco, G., Martínez-Aguilar, K., \& Alvarez-Venegas, R. (2017). Transgenerational defense priming for crop protection against plant pathogens: a hypothesis. Frontiers in plant science, 8, 696.

Remy, J. J. (2010). Stable inheritance of an acquired behavior in Caenorhabditis elegans. Current Biology, 20(20), R877-R878.

Reza Rahavi, M., Migicovsky, Z. D., Titov, V., \& Kovalchuk, I. (2011). Transgenerational adaptation to heavy metal salts in Arabidopsis. Frontiers in plant science, 2, 91.

Richards, E. J. (2008). Population epigenetics. Current opinion in genetics \& development, $18(2), 221-226$.

Schmitz, R. J., Schultz, M. D., Lewsey, M. G., O'Malley, R. C., Urich, M. A., Libiger, O., . . . \& Ecker, J. R. (2011). Transgenerational Epigenetic Instability Is a Source of Novel Methylation Variants. Science, 334(6054), 369-373.

Schultz, M. D., Schmitz, R. J., \& Ecker, J. R. (2012). 'Leveling' the playing field for analyses of single-base resolution DNA methylomes. Trends in Genetics, 28(12), 583-585.

Shastry, B. S. (2009). SNPs: impact on gene function and phenotype. In Single Nucleotide Polymorphisms (pp. 3-22). Humana Press, Totowa, NJ.

Stroud, H., Greenberg, M. V., Feng, S., Bernatavichute, Y. V., \& Jacobsen, S. E. (2013). Comprehensive analysis of silencing mutants reveals complex regulation of the Arabidopsis methylome. Cell, 152(1-2), 352-364.

Sung, D.-Y., Kaplan, F., Lee, K.-J., \& Guy, C. L. (2003). Acquired tolerance to temperature extremes. Trends in plant science, 8(4), 179-187.

Susan, J. C., Harrison, J., Paul, C. L., \& Frommer, M. (1994). High sensitivity mapping of methylated cytosines. Nucleic Acids Research, 22(15), 2990-2997.

Suter, L., \& Widmer, A. (2013a). Phenotypic effects of salt and heat stress over three generations in Arabidopsis thaliana. PloS one, 8(11), e80819.

Suter, L., \& Widmer, A. (2013b). Environmental heat and salt stress induce transgenerational phenotypic changes in arabidopsis thaliana. PloS one, 8(4), e60364.

Thiebaut, F., Hemerly, A. S., \& Ferreira, P. C. G. (2019). A role for epigenetic regulation in the adaptation and stress responses of non-model plants. Frontiers in plant science, 10, 246.

Uthup, T. K., Ravindran, M., Bini, K., \& Thakurdas, S. (2011). Divergent DNA Methylation Patterns Associated with Abiotic Stress in Hevea brasiliensis. Molecular Plant, 4(6), 9961013.

Verhoeven, K. J., \& van Gurp, T. P. (2012). Transgenerational effects of stress exposure on offspring phenotypes in apomictic dandelion. PloS one, 7(6), e38605. 
Vidal, R. O., do Nascimento, L. C., Mondego, J. M. C., Pereira, G. A. G., \& Carazzolle, M. F. (2012). Identification of SNPs in RNA-seq data of two cultivars of Glycine max (soybean) differing in drought resistance. Genetics and Molecular Biology, 35(1 Suppl), 331-334.

Wang, T., Ye, C., Wang, M., \& Chu, G. (2017). Identification of cold-stress responsive proteins in Anabasis aphylla seedlings via the iTRAQ proteomics technique. Journal of Plant Interactions, 12(1), 505-519.

Wang, W., Qin, Q., Sun, F., Wang, Y., Xu, D., Li, Z., \& Fu, B. (2016). Genome-wide differences in DNA methylation changes in two contrasting rice genotypes in response to drought conditions. Frontiers in plant science, 7, 1675.

Wei, T., Wang, Y., \& Liu, J. H. (2020). Comparative transcriptome analysis reveals synergistic and disparate defense pathways in the leaves and roots of trifoliate orange (Poncirus trifoliata) autotetraploids with enhanced salt tolerance. Horticulture Research, 7(1), $1-14$.

Whittle, C., Otto, S., Johnston, M. O., \& Krochko, J. (2009). Adaptive epigenetic memory of ancestral temperature regime in Arabidopsis thaliana. Botany, 87(6), 650-657.

Wibowo, A., Becker, C., Marconi, G., Durr, J., Price, J., Hagmann, J., ... \& Weigel, D. (2016). Hyperosmotic stress memory in Arabidopsis is mediated by distinct epigenetically labile sites in the genome and is restricted in the male germline by DNA glycosylase activity. Elife, 5, e13546.

Xu, J., Zhou, S., Gong, X., Song, Y., van Nocker, S., Ma, F., \& Guan, Q. (2018). Single $\square$ base methylome analysis reveals dynamic epigenomic differences associated with water deficit in apple. Plant biotechnology journal, 16(2), 672-687.

Yadav, N. S., Shukla, P. S., Jha, A., Agarwal, P. K., \& Jha, B. (2012). The SbSOS1 gene from the extreme halophyte Salicornia brachiata enhances $\mathrm{Na}^{+}$loading in xylem and confers salt tolerance in transgenic tobacco. BMC plant biology, 12(1), 188.

Yang, X., Sanchez, R., Kundariya, H., Maher, T., Dopp, I., Schwegel, R., ... \& Mackenzie, S. A. (2020). Segregation of an MSH1 RNAi transgene produces heritable non-genetic memory in association with methylome reprogramming. Nature communications, 11(1), 1-17.

Yao, Y., Bilichak, A., Golubov, A., \& Kovalchuk, I. (2012). ddm1 plants are sensitive to methyl methane sulfonate and $\mathrm{NaCl}$ stresses and are deficient in DNA repair. Plant cell reports, 31(9), 1549-1561.

Zhang, B., Tieman, D. M., Jiao, C., Xu, Y., Chen, K., Fei, Z., ... \& Klee, H. J. (2016). Chilling-induced tomato flavor loss is associated with altered volatile synthesis and transient changes in DNA methylation. Proceedings of the National Academy of Sciences, 113(44), $12580-12585$.

Zhang, H., Lang, Z., \& Zhu, J. K. (2018). Dynamics and function of DNA methylation in plants. Nature reviews Molecular cell biology, 19(8), 489-506.

Zhang, H., Mao, X., Zhang, J., Chang, X., \& Jing, R. (2013). Single-nucleotide polymorphisms and association analysis of drought-resistance gene TaSnRK2. 8 in common wheat. Plant physiology and biochemistry, 70, 174-181. 
Zheng, X., Chen, L., Li, M., Lou, Q., Xia, H., Wang, P., ... \& Luo, L. (2013). Transgenerational variations in DNA methylation induced by drought stress in two rice varieties with distinguished difference to drought resistance. PloS one, 8(11), e80253.

Zheng, X., Chen, L., Xia, H., Wei, H., Lou, Q., Li, M., ... \& Luo, L. (2017). Transgenerational epimutations induced by multi-generation drought imposition mediate rice plant's adaptation to drought condition. Scientific Reports, 7(1), 1-13. 